\title{
Organically Structured Control of Large-Scale Systems with Expanding Construction Based on State Observation
}

\author{
Li Xiaohua, ${ }^{1}$ Liu Xiaoping, ${ }^{2}$ Liu Yang, ${ }^{1}$ and Gao Jiawang ${ }^{1}$ \\ ${ }^{1}$ School of Electronics and Information Engineering, University of Science and Technology Liaoning, Anshan, Liaoning 114051, China \\ ${ }^{2}$ Department of Electrical Engineering, Lakehead University, Thunder Bay, ON, Canada P7B 5E1 \\ Correspondence should be addressed to Li Xiaohua; lixiaohua6412@163.com
}

Received 15 August 2014; Accepted 7 September 2014

Academic Editor: Sakthivel Rathinasamy

Copyright (c) $2015 \mathrm{Li}$ Xiaohua et al. This is an open access article distributed under the Creative Commons Attribution License, which permits unrestricted use, distribution, and reproduction in any medium, provided the original work is properly cited.

\begin{abstract}
This paper presents a new method on the problem of organically structured control based on state observation for a class of largescale systems with expanding construction. This problem is to design a local state feedback controller and an observer for a new subsystem which is added to a large-scale system without changing the decentralized state feedback control laws of the original construction, so that both the new subsystem and the resulting expanded system are robustly connectively stable. Firstly, based on state observers, the mathematical model of a large-scale system with expanding construction is reestablished and analyzed. In addition, the sufficient condition for robust decentralized connective stabilization of the expanded construction of large-scale systems is deduced by taking an LMI approach, which is further relaxed by removing the square matrix condition on the output matrix. This problem is transformed into solving an LMI problem. The new design method of an organically structured controller and observer for the expanded construction is also given. Finally, the simulation examples show the effectiveness of the proposed method.
\end{abstract}

\section{Introduction}

Structural changes of large-scale interconnected systems occur in real world applications, which have a negative impact on stability of the systems. The question of how to reduce these negative effects attracts close attention from many scholars [1-4]. Siljak, who studied these stability problems from the viewpoint of structural perturbations, proposed the concept of connective stability [1] and introduced the idea of organically structured control at a bionic angle [2]. Up to now, structural changes, such as situations in which some subsystems may be disconnected and then reconnected, have been considered in research, and organically structured control methods have been given accordingly. Therefore, structural control of large-scale interconnected systems has become one of new research topics. However, the abovementioned structural changes do not cover all cases, such as expanded construction in which new systems are added to an original structure. The expanded construction, which is often encountered, was first proposed in [5]. It discussed the decentralized control problem by taking a frequency domain approach. Due to the complexity of the frequency domain design, $[6,7]$ provided sufficient conditions for the solvability of the robust decentralized connective stabilization problem by taking LMI theory and proposed an organically structured control method for the expanded construction of large-scale systems. However, all the existing results are based on state feedback. Since not all states are measurable, a state observer is necessary. Researches on state estimation and the observer-based decentralized control design have been well investigated in literature; see [8-13] and the references therein. The observer-based decentralized controller was derived for large-scale systems with expanding construction in [14]. It is required that the output matrix $C$ of the added subsystems be a square matrix, which is very restrictive.

In this paper, a mathematical model is reestablished for a large-scale system with expanding construction. Comparing with [14], an improved method is proposed for designing an organically structured controller and observer of 


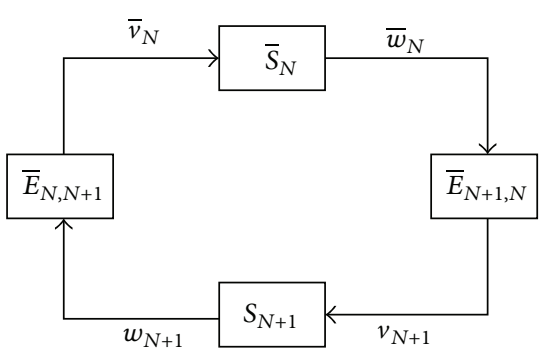

FIgURE 1: The basic structure of an expanded system.

the expanded construction. A new sufficient condition is obtained for solving the observer-based decentralized control design problem. Unlike [14], the restrictive condition on the output matrix $C$ is no longer necessary. Some simulations are given for a power system with expanding construction to show the effectiveness of the proposed method.

The main contributions are as follows. (1) An observerbased decentralized feedback controller is designed for a class of large-scale systems with expanding structure. (2) A sufficient condition for the existence of such controller is derived, which is less restrictive than that in [14]. (3) A LMIbased design approach is proposed to design such controller, which is simpler than the frequency-domain method in [5]. (4) Such design method can be easily implemented for only the newly added subsystem without changing the controllers for the original subsystems no matter what design method was used in the controller design for the original subsystems.

\section{Mathematical Model of Large-Scale Systems with Expanding Construction Based on State Observation}

Consider a class of large-scale systems with expanding construction as in [5]. The basic structure of these systems is shown in Figure 1.

In Figure $1, \bar{S}_{N}$ is the original system structure which is composed of $N$ subsystems, and $S_{N+1}$ is the $(N+1)$ th subsystem subsequently added to $\bar{S}_{N} \cdot \bar{v}_{N}=\left(v_{1}, v_{2}, \ldots, v_{N}\right)^{\mathrm{T}}$ and $\bar{w}_{N}=\left(w_{1}, w_{2}, \ldots, w_{N}\right)^{\mathrm{T}}$, where $v_{i}$ is the variable which denotes the interconnection term of the $i$ th subsystem from the other subsystems, $i=1,2, \ldots, N+1$, while $w_{i}$ is the variable which represents the impact on the other subsystems by the $i$ th subsystem. The expanded system includes $N+1$ subsystems in total. The connective relations between the subsystems are expressed by the connective matrix $E=\left(E_{i, j}\right)$, where $E_{i, j}$ represents the interconnection from the $j$ th subsystem to the $i$ th subsystem. $E_{i, j}=1$ represents the fact that there is an interconnection and $E_{i, j}=0$ means that there is no interconnection. The connective relations of the original system structure can be described as

$$
\bar{E}_{N}=\left[\begin{array}{cccc}
0 & E_{1,2} & \cdots & E_{1, N} \\
E_{2,1} & 0 & \cdots & E_{2, N} \\
\vdots & \vdots & \ddots & \vdots \\
E_{N, 1} & E_{N, 2} & \cdots & 0
\end{array}\right]
$$

In Figure 1, $\bar{E}_{N, N+1}=\left[E_{1, N+1}^{\mathrm{T}}, E_{2, N+1}^{\mathrm{T}}, \ldots, E_{N, N+1}^{\mathrm{T}}\right]^{\mathrm{T}}$ denotes the new column of the interconnected matrix after the new subsystem is added, while $\bar{E}_{N+1, N}=\left[E_{N+1,1}, E_{N+1,2}\right.$, $\left.\ldots, E_{N+1, N}\right]$ represents the new row. The new interconnected matrix is

$$
\bar{E}_{N+1}=\left[\begin{array}{ccccc}
0 & E_{1,2} & \cdots & E_{1, N} & E_{1, N+1} \\
E_{2,1} & 0 & \cdots & E_{2, N} & E_{2, N+1} \\
\vdots & \vdots & \ddots & \vdots & \vdots \\
E_{N, 1} & E_{N, 2} & \cdots & 0 & E_{N, N+1} \\
E_{N+1,1} & E_{N+1,2} & \cdots & E_{N+1, N} & 0
\end{array}\right] .
$$

Consider $N$ subsystems in the original structure, which are controlled by state feedback with state observers. The model of the structure is described as

$$
\begin{aligned}
\dot{x}_{i} & =A_{i} x_{i}+B_{i} u_{i}+G_{i} v_{i}, \\
y_{i} & =C_{i} x_{i}, \\
w_{i} & =H_{i} x_{i}, \\
\dot{\tilde{x}}_{i} & =A_{i} \tilde{x}_{i}+B_{i} u_{i}+L_{i}\left(C_{i} x_{i}-C_{i} \tilde{x}_{i}\right), \\
u_{i} & =K_{i} \tilde{x}_{i},
\end{aligned}
$$

with $i=1,2, \ldots, N$ and static interconnections as follows:

$$
v_{i}=\sum_{j=1, j \neq i}^{N} E_{i, j} w_{j},
$$

where $x_{i}$ is the state vector of the $i$ th subsystem, $\tilde{x}_{i}$ is the state observer vector, $u_{i}$ is the control input vector, and $y_{i}$ is the output vector. $A_{i}, B_{i}, C_{i}, G_{i}, H_{i}, K_{i}$, and $L_{i}$ are the constant matrices with appropriate dimensions.

Setting $e_{i}=x_{i}-\tilde{x}_{i}$, the $i$ th subsystem can then be written as

$$
\begin{aligned}
& \dot{x}_{i}=\left(A_{i}+B_{i} K_{i}\right) x_{i}-B_{i} K_{i} e_{i}+G_{i} v_{i}, \\
& \dot{e}_{i}=\left(A_{i}-L_{i} C_{i}\right) e_{i}+G_{i} v_{i},
\end{aligned}
$$

with the connective relations given in (4). Therefore, the mathematical description of the $N$ close-loop subsystems can be denoted as $\bar{S}_{N}$. Consider

$$
\begin{aligned}
{\left[\begin{array}{c}
\dot{\bar{X}}_{N} \\
\dot{\bar{e}}_{N}
\end{array}\right]=} & {\left[\begin{array}{cc}
\bar{A}_{N}+\bar{B}_{N} \bar{K}_{N}+\bar{G}_{N} \bar{E}_{N} \bar{H}_{N}-\bar{B}_{N} \bar{K}_{N} \\
\bar{G}_{N} \bar{E}_{N} \bar{H}_{N} & \bar{A}_{N}-\bar{L}_{N} \bar{C}_{N}
\end{array}\right] } \\
& \times\left[\begin{array}{c}
\bar{X}_{N} \\
\bar{e}_{N}
\end{array}\right], \\
\bar{y}_{N}= & \bar{C}_{N} \bar{X}_{N},
\end{aligned}
$$


where $\bar{X}_{N}=\left(x_{1}^{\mathrm{T}}, x_{2}^{\mathrm{T}}, \ldots, x_{N}^{\mathrm{T}}\right)^{\mathrm{T}}$ is the state vector of the original system, $\bar{e}_{N}=\left[e_{1}^{\mathrm{T}}, e_{2}^{\mathrm{T}}, \ldots, e_{N}^{\mathrm{T}}\right]^{\mathrm{T}}$ is the error vector, and $\bar{y}_{N}=\left(y_{1}^{\mathrm{T}}, y_{2}^{\mathrm{T}}, \ldots, y_{N}^{\mathrm{T}}\right)^{\mathrm{T}}$ is the output. The matrices $\bar{A}_{N}, \bar{B}_{N}$, $\bar{C}_{N}, \bar{G}_{N}$, and $\bar{H}_{N}$ are defined as

$$
\begin{aligned}
& \bar{A}_{N}=\operatorname{diag}\left(A_{1}, A_{2}, \ldots, A_{N}\right), \\
& \bar{B}_{N}=\operatorname{diag}\left(B_{1}, B_{2}, \ldots, B_{N}\right), \\
& \bar{C}_{N}=\operatorname{diag}\left(C_{1}, C_{2}, \ldots, C_{N}\right), \\
& \bar{G}_{N}=\operatorname{diag}\left(G_{1}, G_{2}, \ldots, G_{N}\right), \\
& \bar{H}_{N}=\operatorname{diag}\left(H_{1}, H_{2}, \ldots, H_{N}\right) .
\end{aligned}
$$

$\bar{K}_{N}=\operatorname{diag}\left(K_{1}, K_{2}, \ldots, K_{N}\right)$ is the controller gain matrix of the original system. $\bar{L}_{N}=\operatorname{diag}\left(L_{1}, L_{2}, \ldots, L_{N}\right)$ is the observer gain matrix of the original system.

Suppose that a new subsystem is added to the original system $\bar{S}_{N}$. Due to the newly added interconnections, the mathematical description of the original closed-loop system (6) can be modified as follows:

$$
\begin{aligned}
{\left[\begin{array}{c}
\dot{\bar{X}}_{N} \\
\dot{\bar{e}}_{N}
\end{array}\right]=} & {\left[\begin{array}{cc}
\bar{A}_{N}+\bar{B}_{N} \bar{K}_{N}+\bar{G}_{N} \bar{E}_{N} \bar{H}_{N} & -\bar{B}_{N} \bar{K}_{N} \\
\bar{G}_{N} \bar{E}_{N} \bar{H}_{N} & \bar{A}_{N}-\bar{L}_{N} \bar{C}_{N}
\end{array}\right]\left[\begin{array}{l}
\bar{X}_{N} \\
\bar{e}_{N}
\end{array}\right] } \\
& +\left[\begin{array}{l}
\bar{G}_{N} \\
\bar{G}_{N}
\end{array}\right] \bar{v}_{N}, \\
\bar{w}_{N}= & {\left[\begin{array}{ll}
\bar{H}_{N} & 0
\end{array}\right]\left[\begin{array}{l}
\bar{X}_{N} \\
\overline{\bar{X}}_{N}
\end{array}\right], } \\
\bar{y}_{N}= & {\left[\begin{array}{ll}
\bar{C}_{N} & 0
\end{array}\right]\left[\begin{array}{l}
\bar{X}_{N} \\
\overline{\bar{X}}_{N}
\end{array}\right] . }
\end{aligned}
$$

Suppose that the model of $(N+1)$ th subsystem $\left(S_{N+1}\right)$ is described by (3) with $i=N+1$. The connective relationships between the newly added subsystem and the original system can be expressed as

$$
\bar{v}_{N}=\bar{E}_{N, N+1} w_{N+1}, \quad v_{N+1}=\bar{E}_{N+1, N} \bar{w}_{N} .
$$

The original system and the newly added subsystem are combined together to get the following closed-loop system. Consider the following:

$$
\begin{aligned}
{\left[\begin{array}{c}
\dot{\bar{X}}_{N} \\
\dot{\bar{e}}_{N} \\
\dot{x}_{N+1} \\
\dot{e}_{N+1}
\end{array}\right]=} & {\left[\begin{array}{cccc}
\bar{A}_{N}+\bar{B}_{N} \bar{K}_{N} & -\bar{B}_{N} \bar{K}_{N} & \mathbf{0} & \mathbf{0} \\
0 & \bar{A}_{N}-\bar{L}_{N} \bar{C}_{N} & \mathbf{0} & \mathbf{0} \\
\mathbf{0} & \mathbf{0} & A_{N+1}+B_{N+1} K_{N+1} & -B_{N+1} K_{N+1} \\
\mathbf{0} & \mathbf{0} & \mathbf{0} & A_{N+1}-L_{N+1} C_{N+1}
\end{array}\right]\left[\begin{array}{c}
\bar{X}_{N} \\
\bar{e}_{N} \\
x_{N+1} \\
e_{N+1}
\end{array}\right] } \\
& +\left[\begin{array}{cccc}
\bar{G}_{N} \bar{E}_{N} \bar{H}_{N} & \mathbf{0} \bar{G}_{N} \bar{E}_{N, N+1} H_{N+1} & \mathbf{0} \\
\bar{G}_{N} \bar{E}_{N} \bar{H}_{N} & \mathbf{0} \bar{G}_{N} \bar{E}_{N, N+1} H_{N+1} & \mathbf{0} \\
G_{N+1} \bar{E}_{N+1, N} \bar{H}_{N} & \mathbf{0} & \mathbf{0} & \mathbf{0} \\
G_{N+1} \bar{E}_{N+1, N} \bar{H}_{N} & \mathbf{0} & \mathbf{0} & \mathbf{0}
\end{array}\right]\left[\begin{array}{c}
\bar{X}_{N} \\
\bar{e}_{N} \\
x_{N+1} \\
e_{N+1}
\end{array}\right],
\end{aligned}
$$

which can be rewritten as

$$
\begin{aligned}
\dot{X} & =\left[\begin{array}{cc}
\widehat{A}_{N} & \mathbf{0} \\
\mathbf{0} & \widehat{A}_{N+1}
\end{array}\right] X+h(t, X, E) \\
& =A X+h(t, X, E),
\end{aligned}
$$

where

$$
\begin{gathered}
X=\left[\bar{X}_{N}^{\mathrm{T}}, \bar{e}_{N}^{\mathrm{T}}, x_{N+1}^{\mathrm{T}}, e_{N+1}^{\mathrm{T}}\right]^{\mathrm{T}}, \\
\widehat{A}_{N}=\left[\begin{array}{cc}
\bar{A}_{N}+\bar{B}_{N} \bar{K}_{N} & -\bar{B}_{N} \bar{K}_{N} \\
\mathbf{0} & \bar{A}_{N}-\bar{L}_{N} \bar{C}_{N}
\end{array}\right],
\end{gathered}
$$




\section{Organically Structured Control Design of a System with Expanding Construction}

Since the addition of new subsystems occurs during the operation of the original construction, it is more realistic to keep the decentralized control laws of the original subsystems unchanged. For this reason, it is necessary that the control law of the new subsystem is able to stabilize connectively both itself and the resultant large-scale system without changing the original decentralized control laws. Therefore, organically structured control of large-scale systems with expanding construction requires the control law of the newly added subsystem to be designed separately. Here, we first define the concept of connective stability and organically structured control.

Definition 1 (see [1]). A large-scale interconnected system is connectively stable if the equilibrium state of the system is asymptotically stable when all structural perturbations take place. The structural perturbations include the following two cases. Case 1: new subsystems are added to the original construction. Case 2: some subsystems are disconnected from the large-scale system and then reconnected.

As a matter of fact, the problem of organically structured control of the interconnected system is to ensure that the decentralized control laws make the system connectively stable when the system structure is reconstructed. In this problem, an interconnected system can be treated as an organism and thus is called organically structured control.

Definition 2 (see [2]). For an interconnected system including a certain number of subsystems, the organically structured control problem is to design a decentralized control law $u_{i}$ for each subsystem, so that the closed-loop system is connectively stable when the system structure is reconstructed.

The main result of this paper is as follows.

Theorem 3. The expanded system (11) with state observers can be robustly connectively stabilized when $(N+1)$ th subsystem with a state observer is added to the original system structure if there are symmetrical positive definite matrices $\bar{P}_{N}>0, P_{N+1}>0$ as well as the matrices $M_{N+1}, N_{N+1}$ and interconnected constraint matrices $\bar{q}_{1}, \bar{q}_{2}, \bar{q}_{3}$, so that the problem

\section{minimize $\gamma$}

$$
\left[\begin{array}{cccccccc}
\Lambda_{N} & \mathbf{0} & \mathbf{0} & \bar{P}_{N} & \mathbf{0} & \mathbf{0} & \bar{q}_{1}^{\mathrm{T}} & \bar{q}_{3}^{\mathrm{T}} \\
\mathbf{0} & \widetilde{\Lambda}_{N+1,1} & \widetilde{\Lambda}_{N+1,2} & \mathbf{0} & I & \mathbf{0} & Y_{N+1,1} \bar{q}_{2}^{\mathrm{T}} & \mathbf{0} \\
\mathbf{0} & \widetilde{\Lambda}_{N+1,3} & \Lambda_{N+1,4} & \mathbf{0} & \mathbf{0} & P_{N+1,2} & \mathbf{0} & \mathbf{0} \\
\bar{P}_{N} & \mathbf{0} & \mathbf{0} & -\tau I & \mathbf{0} & \mathbf{0} & \mathbf{0} & \mathbf{0} \\
\mathbf{0} & I & \mathbf{0} & \mathbf{0} & -\tau I & \mathbf{0} & \mathbf{0} & \mathbf{0} \\
\mathbf{0} & \mathbf{0} & P_{N+1,2} & \mathbf{0} & \mathbf{0} & -\tau I & \mathbf{0} & \mathbf{0} \\
\bar{q}_{1} & \bar{q}_{2} Y_{N+1,1} & \mathbf{0} & \mathbf{0} & \mathbf{0} & \mathbf{0} & -\gamma I & \mathbf{0} \\
\bar{q}_{3} & \mathbf{0} & \mathbf{0} & \mathbf{0} & \mathbf{0} & \mathbf{0} & \mathbf{0} & -\gamma I
\end{array}\right]
$$

is feasible, where

$$
\begin{aligned}
\widetilde{\Lambda}_{N+1,1}= & Y_{N+1,1} A_{N+1}^{\mathrm{T}}+A_{N+1} Y_{N+1,1}+M_{N+1}^{\mathrm{T}} B_{N+1}^{\mathrm{T}} \\
& +B_{N+1} M_{N+1}, \\
\widetilde{\Lambda}_{N+1,2}= & -B_{N+1} K_{N+1}, \\
\widetilde{\Lambda}_{N+1,3}= & -K_{N+1}^{\mathrm{T}} B_{N+1}^{\mathrm{T}}, \\
\Lambda_{N+1,4}= & A_{N+1}^{\mathrm{T}} P_{N+1,2}+P_{N+1,2} A_{N+1}-C_{N+1}^{\mathrm{T}} N_{N+1}^{\mathrm{T}} \\
& -N_{N+1} C_{N+1} .
\end{aligned}
$$

The control law and observer gain of the newly added subsystem can be determined by

$$
K_{N+1}=M_{N+1} Y_{N+1,1}^{-1}, \quad L_{N+1}=P_{N+1,2}^{-1} N_{N+1} .
$$

Proof. To design a controller and observer for organically structured control of the expanded system, let us choose a Lyapunov function $V(X)=X^{\mathrm{T}} P X$ with

$$
\begin{aligned}
P & =\left[\begin{array}{cccc}
\bar{P}_{N, 1} & \mathbf{0} & \mathbf{0} & \mathbf{0} \\
\mathbf{0} & \bar{P}_{N, 2} & \mathbf{0} & \mathbf{0} \\
\mathbf{0} & \mathbf{0} & P_{N+1,1} & \mathbf{0} \\
\mathbf{0} & \mathbf{0} & \mathbf{0} & P_{N+1,2}
\end{array}\right] \\
& =\left[\begin{array}{cc}
\bar{P}_{N} & \mathbf{0} \\
\mathbf{0} & P_{N+1}
\end{array}\right]>0 .
\end{aligned}
$$

Then

$$
\dot{V}(X)=X^{\mathrm{T}}\left(A^{\mathrm{T}} P+P A\right) X+h^{\mathrm{T}} P X+X^{\mathrm{T}} P h .
$$

If the system is stable, (17) is equivalent to

$$
\begin{gathered}
P>0 \\
{\left[\begin{array}{c}
X \\
h
\end{array}\right]^{\mathrm{T}}\left[\begin{array}{cc}
A^{\mathrm{T}} P+P A & P \\
P & \mathbf{0}
\end{array}\right]\left[\begin{array}{l}
X \\
h
\end{array}\right]<0 .}
\end{gathered}
$$

Consider that the structural perturbation in (11) is bounded quadratically; that is,

$$
h^{\mathrm{T}}(t, X, E) h(t, X, E) \leq \alpha^{2} X^{\mathrm{T}} Q^{\mathrm{T}} \mathrm{Q} X,
$$

which is equivalent to the following matrix inequality:

$$
\left[\begin{array}{l}
X \\
h
\end{array}\right]^{\mathrm{T}}\left[\begin{array}{cc}
-\alpha^{2} Q^{\mathrm{T}} Q & \mathbf{0} \\
\mathbf{0} & I
\end{array}\right]\left[\begin{array}{l}
X \\
h
\end{array}\right] \leq 0,
$$

where $\alpha$ is the bounding parameter for the uncertain interconnection term of the expanded system and $Q$ is the interconnected constraint matrix given as

$$
\begin{gathered}
Q=\left[\begin{array}{llll}
q_{1} & \mathbf{0} & q_{2} & \mathbf{0} \\
q_{1} & \mathbf{0} & q_{2} & \mathbf{0} \\
q_{3} & \mathbf{0} & \mathbf{0} & \mathbf{0} \\
q_{3} & \mathbf{0} & \mathbf{0} & \mathbf{0}
\end{array}\right]=\left[\begin{array}{ccc}
\bar{q}_{1} & \bar{q}_{2} & \mathbf{0} \\
\bar{q}_{3} & \mathbf{0} & \mathbf{0}
\end{array}\right], \\
\bar{q}_{1}=\left[\begin{array}{ll}
q_{1} & \mathbf{0} \\
q_{1} & \mathbf{0}
\end{array}\right], \quad \bar{q}_{2}=\left[\begin{array}{l}
q_{2} \\
q_{2}
\end{array}\right], \quad \bar{q}_{3}=\left[\begin{array}{ll}
q_{3} & \mathbf{0} \\
q_{3} & \mathbf{0}
\end{array}\right] .
\end{gathered}
$$


Using S-procedure to (18) and (20) and Schur complement lemma, the following inequality is obtained:

$$
\begin{gathered}
P>0 \\
{\left[\begin{array}{ccc}
A^{\mathrm{T}} P+P A & P & Q^{\mathrm{T}} \\
P & -\tau I & \mathbf{0} \\
Q & \mathbf{0} & -\gamma I
\end{array}\right]<0,}
\end{gathered}
$$

with $\tau>0$ and $\gamma=1 / \tau \alpha^{2}$.

It is apparent that $\bar{P}_{N}$ and $P_{N+1}$ are positive definite and symmetrical matrices. The dimensions of $\bar{P}_{N}$ are the same as the original-construction system with observers and the dimensions of $P_{N+1}$ are the same as the newly added subsystem with observer. The original-construction subsystems need not be designed, so only the newly added subsystem needs to be designed. Then, substituting (11) for $A$ into (22), we can obtain

$$
A^{\mathrm{T}} P+P A=\left[\begin{array}{ccc}
\hat{A}_{N}^{\mathrm{T}} \bar{P}_{N}+\bar{P}_{N} \widehat{A}_{N} & \mathbf{0} & \mathbf{0} \\
\mathbf{0} & \Lambda_{N+1,1} & \Lambda_{N+1,2} \\
\mathbf{0} & \Lambda_{N+1,3} & \Lambda_{N+1,4}
\end{array}\right]
$$

with

$$
\begin{aligned}
\Lambda_{N+1,1}= & A_{N+1}^{\mathrm{T}} P_{N+1,1}+P_{N+1,1} A_{N+1}+K_{N+1}^{\mathrm{T}} B_{N+1}^{\mathrm{T}} P_{N+1,1} \\
& +P_{N+1,1} B_{N+1} K_{N+1}, \\
\Lambda_{N+1,2}= & -P_{N+1,1} B_{N+1} K_{N+1}, \\
\Lambda_{N+1,3}= & -K_{N+1}^{\mathrm{T}} B_{N+1}^{\mathrm{T}} P_{N+1,1}, \\
\Lambda_{N+1,4}= & A_{N+1}^{\mathrm{T}} P_{N+1,2}+P_{N+1,2} A_{N+1}-C_{N+1}^{\mathrm{T}} L_{N+1}^{\mathrm{T}} P_{N+1,2} \\
& -P_{N+1,2} L_{N+1} C_{N+1} .
\end{aligned}
$$

Therefore, (22) can be written as

$$
\left[\begin{array}{cccccccc}
\Lambda_{N} & \mathbf{0} & \mathbf{0} & \bar{P}_{N} & \mathbf{0} & \mathbf{0} & \bar{q}_{1}^{\mathrm{T}} & \bar{q}_{3}^{\mathrm{T}} \\
\mathbf{0} & \Lambda_{N+1,1} & \Lambda_{N+1,2} & \mathbf{0} & P_{N+1,1} & \mathbf{0} & \bar{q}_{2}^{\mathrm{T}} & \mathbf{0} \\
\mathbf{0} & \Lambda_{N+1,3} & \Lambda_{N+1,4} & \mathbf{0} & \mathbf{0} & P_{N+1,2} & \mathbf{0} & \mathbf{0} \\
\bar{P}_{N} & \mathbf{0} & \mathbf{0} & -\tau I & \mathbf{0} & \mathbf{0} & \mathbf{0} & \mathbf{0} \\
\mathbf{0} & P_{N+1,1} & \mathbf{0} & \mathbf{0} & -\tau I & \mathbf{0} & \mathbf{0} & \mathbf{0} \\
\mathbf{0} & \mathbf{0} & P_{N+1,2} & \mathbf{0} & \mathbf{0} & -\tau I & \mathbf{0} & \mathbf{0} \\
\bar{q}_{1} & \bar{q}_{2} & \mathbf{0} & \mathbf{0} & \mathbf{0} & \mathbf{0} & -\gamma I & \mathbf{0} \\
\bar{q}_{3} & \mathbf{0} & \mathbf{0} & \mathbf{0} & \mathbf{0} & \mathbf{0} & \mathbf{0} & -\gamma I
\end{array}\right]<0,
$$

with $\Lambda_{N}=\widehat{\bar{A}}_{N}^{\mathrm{T}} \bar{P}_{N}+\bar{P}_{N} \widehat{A}_{N}$.
Set $F=\operatorname{diag}\left(I, P_{N+1,1}^{-1}, I, I, I, I, I, I\right)$ and $Y_{N+1,1}=P_{N+1,1}^{-1}$. Pre- and postmultiplying (25) by $F$ gives

$$
\begin{aligned}
& F\left[\begin{array}{cccccccc}
\Lambda_{N} & \mathbf{0} & \mathbf{0} & \bar{P}_{N} & \mathbf{0} & \mathbf{0} & \bar{q}_{1}^{\mathrm{T}} & \bar{q}_{3}^{\mathrm{T}} \\
\mathbf{0} & \Lambda_{N+1,1} & \Lambda_{N+1,2} & \mathbf{0} & P_{N+1,1} & \mathbf{0} & \bar{q}_{2}^{\mathrm{T}} & \mathbf{0} \\
\mathbf{0} & \Lambda_{N+1,3} & \Lambda_{N+1,4} & \mathbf{0} & \mathbf{0} & P_{N+1,2} & \mathbf{0} & \mathbf{0} \\
\bar{P}_{N} & \mathbf{0} & \mathbf{0} & -\tau I & \mathbf{0} & \mathbf{0} & \mathbf{0} & \mathbf{0} \\
\mathbf{0} & P_{N+1,1} & \mathbf{0} & \mathbf{0} & -\tau I & \mathbf{0} & \mathbf{0} & \mathbf{0} \\
\mathbf{0} & \mathbf{0} & P_{N+1,2} & \mathbf{0} & \mathbf{0} & -\tau I & \mathbf{0} & \mathbf{0} \\
\bar{q}_{1} & \bar{q}_{2} & \mathbf{0} & \mathbf{0} & \mathbf{0} & \mathbf{0} & -\gamma I & \mathbf{0} \\
\bar{q}_{3} & \mathbf{0} & \mathbf{0} & \mathbf{0} & \mathbf{0} & \mathbf{0} & \mathbf{0} & -\gamma I
\end{array}\right] F \\
& <0
\end{aligned}
$$

so that the following inequality can be obtained:

$$
\begin{gathered}
{\left[\begin{array}{cccccccc}
\Lambda_{N} & \mathbf{0} & \mathbf{0} & \bar{P}_{N} & \mathbf{0} & \mathbf{0} & \bar{q}_{1}^{\mathrm{T}} & \bar{q}_{3}^{\mathrm{T}} \\
\mathbf{0} & \widetilde{\Lambda}_{N+1,1} & \widetilde{\Lambda}_{N+1,2} & \mathbf{0} & I & \mathbf{0} & Y_{N+1,1} \bar{q}_{2}^{\mathrm{T}} & \mathbf{0} \\
\mathbf{0} & \widetilde{\Lambda}_{N+1,3} & \Lambda_{N+1,4} & \mathbf{0} & \mathbf{0} & P_{N+1,2} & \mathbf{0} & \mathbf{0} \\
\bar{P}_{N} & \mathbf{0} & \mathbf{0} & -\tau I & \mathbf{0} & \mathbf{0} & \mathbf{0} & \mathbf{0} \\
\mathbf{0} & I & \mathbf{0} & \mathbf{0} & -\tau I & \mathbf{0} & \mathbf{0} & \mathbf{0} \\
\mathbf{0} & \mathbf{0} & P_{N+1,2} & \mathbf{0} & \mathbf{0} & -\tau I & \mathbf{0} & \mathbf{0} \\
\bar{q}_{1} & \bar{q}_{2} Y_{N+1,1} & \mathbf{0} & \mathbf{0} & \mathbf{0} & \mathbf{0} & -\gamma I & \mathbf{0} \\
\bar{q}_{3} & \mathbf{0} & \mathbf{0} & \mathbf{0} & \mathbf{0} & \mathbf{0} & \mathbf{0} & -\gamma I
\end{array}\right]} \\
<0,
\end{gathered}
$$

where

$$
\begin{aligned}
\widetilde{\Lambda}_{N+1,1}= & Y_{N+1,1} A_{N+1}^{\mathrm{T}}+A_{N+1} Y_{N+1,1}+Y_{N+1,1} K_{N+1}^{\mathrm{T}} B_{N+1}^{\mathrm{T}} \\
& +B_{N+1} K_{N+1} Y_{N+1,1}, \\
\widetilde{\Lambda}_{N+1,2}= & -B_{N+1} K_{N+1}, \\
\widetilde{\Lambda}_{N+1,3}= & -K_{N+1}^{\mathrm{T}} B_{N+1}^{\mathrm{T}} .
\end{aligned}
$$

Note that (27) is not an LMI. However, by setting $K_{N+1} Y_{N+1,1}=M_{N+1}$ and $P_{N+1,2} L_{N+1}=N_{N+1}$ so that

$$
\begin{aligned}
\widetilde{\Lambda}_{N+1,1}= & Y_{N+1,1} A_{N+1}^{\mathrm{T}}+A_{N+1} Y_{N+1,1}+M_{N+1}^{\mathrm{T}} B_{N+1}^{\mathrm{T}} \\
& +B_{N+1} M_{N+1}, \\
\Lambda_{N+1,4}= & A_{N+1}^{\mathrm{T}} P_{N+1,2}+P_{N+1,2} A_{N+1}-C_{N+1}^{\mathrm{T}} N_{N+1}^{\mathrm{T}} \\
& -N_{N+1} C_{N+1},
\end{aligned}
$$

(27) can thus be transformed into an LMI. $K_{N+1}$ and $L_{N+1}$ can be determined by (17).

Because the changes of $E_{i, j}$ in $\bar{E}_{N, N+1}$ and $\bar{E}_{N+1, N}$ from 1 to 0 or 0 to 1 are considered in the constraints of 
the interconnected items, including random situations and $h(t, X, E) \equiv 0$, the results obtained are connectively stable.

Therefore, the theorem has been proved.

Remark 4. The method deduced in this paper can be applied to an expanded subsystem with any dimensionality. In addition, the controllers for the subsystems in the original structure can be permitted to use different control methods, such as LQR and pole-placement. Therefore, the presented method is more practical.

\section{Application to Interconnected Power System Expansion}

Consider a class of multiarea interconnected power systems, in which each area includes a hydroelectric power unit and a thermal power unit. The mathematical model, state variables, and output variables can be found from $[15,16]$. This is a deviation model of automatic generation control (AGC). The $i$ th area-subsystem model $S_{i}$ can be described as

$$
\begin{aligned}
& \dot{x}_{i}=A_{i i} x_{i}+B_{i} u_{i}+\Gamma_{i} \xi_{i}+\sum_{j=1, j \neq i}^{N} A_{i j} x_{j}, \\
& y_{i}=C_{i} x_{i}
\end{aligned}
$$

where $x_{i} \in R^{n_{i}}, u_{i} \in R^{m_{i}}, y_{i} \in R^{l_{i}}, \xi_{i} \in R^{m_{i}}$ are the state, control input, output, and uncertain disturbance input of subsystems, respectively. Consider the following:

$$
\begin{gathered}
A_{i i}=\left[\begin{array}{ccc}
A_{i} & \mathbf{0} & a_{t i} \\
d_{i}^{\mathrm{T}} & \mathbf{0} & 1 \\
\alpha_{1 i} \sum_{j=1, j \neq i}^{N} m_{i j}^{\mathrm{T}} & \mathbf{0} & \mathbf{0}
\end{array}\right], \quad A_{i j}=\left[\begin{array}{cccc}
\mathbf{0} & \mathbf{0} & \mathbf{0} \\
\mathbf{0} & 0 & 0 \\
-\alpha_{1 i} m_{j i}^{\mathrm{T}} & 0 & 0
\end{array}\right], \\
B_{i}=\left[\begin{array}{c}
b_{i} \\
0 \\
0
\end{array}\right], \quad \Gamma_{i}=\left[\begin{array}{c}
f_{i} \\
0 \\
0
\end{array}\right], \quad C_{i}=\left[\begin{array}{ccc}
c_{i} & \mathbf{0} & \mathbf{0} \\
\mathbf{0} & 1 & 0 \\
\mathbf{0} & 0 & 1
\end{array}\right],
\end{gathered}
$$

with

$$
\begin{aligned}
& A_{1}=\left[\begin{array}{cccccccc}
-0.2 & 0 & 0 & 0 & 0 & 0 & 0 & -4 \\
4.75 & -5 & 0 & 0 & 0 & 0 & 0 & 0 \\
0 & 0.1667 & -0.1667 & 0 & 0 & 0 & 0 & 0 \\
0 & 0 & 2 & -2 & 0 & 0 & 0 & 0 \\
0 & -0.08 & -0.0747 & -0.112 & -3.994 & 10 & -0.928 & -9.1011 \\
0 & 0 & 0 & 0 & 0.2 & -0.5 & 0 & 0 \\
0 & 0 & 0 & 0 & 1.3194 & 0 & -1.3889 & -0.2778 \\
0 & 0.01 & 0.0093 & 0.014 & -0.0632 & 0 & 0.116 & -0.1124
\end{array}\right], \\
& a_{t 1}=a_{t 2}=a_{t 3}=\left[\begin{array}{llllllll}
0 & 0 & 0 & 0 & 0.6667 & 0 & 0 & -0.0833
\end{array}\right]^{\mathrm{T}} \text {, } \\
& d_{1}^{\mathrm{T}}=d_{2}^{\mathrm{T}}=\left[\begin{array}{llllllll}
0 & 0 & 0 & 0 & 0 & 0 & 0 & 10
\end{array}\right], \\
& m_{12}^{\mathrm{T}}=m_{21}^{\mathrm{T}}=\left[\begin{array}{llllllll}
0 & 0 & 0 & 0 & 0 & 0 & 0 & 22.2144
\end{array}\right] \text {, } \\
& A_{2}=\left[\begin{array}{cccccccc}
-0.2 & 0 & 0 & 0 & 0 & 0 & 0 & -4 \\
4.75 & -5 & 0 & 0 & 0 & 0 & 0 & 0 \\
0 & 0.1667 & -0.1667 & 0 & 0 & 0 & 0 & 0 \\
0 & 0 & 2 & -2 & 0 & 0 & 0 & 0 \\
0 & -0.1 & -0.0933 & -0.14 & -4.096 & 10 & -0.7442 & -9.1079 \\
0 & 0 & 0 & 0 & 0.2 & -0.5 & 0 & 0 \\
0 & 0 & 0 & 0 & 1.3194 & 0 & -1.3889 & -0.2778 \\
0 & 0.0125 & 0.0117 & 0.0175 & -0.0506 & 0 & 0.0928 & -0.1115
\end{array}\right] \text {, } \\
& A_{3}=A_{2}, \quad C_{3}=C_{2}, \\
& b_{1}=\left[\begin{array}{llllllll}
1.6 & 0 & 0 & 0 & 6 & 0 & 0 & 0
\end{array}\right]^{\mathrm{T}} \text {, } \\
& b_{2}=\left[\begin{array}{llllllll}
2 & 0 & 0 & 0 & 5 & 0 & 0 & 0
\end{array}\right]^{\mathrm{T}} \text {, } \\
& f_{1}=a_{t 1}, \quad f_{2}=a_{t 2}, \quad f_{3}=a_{t 3} \text {, }
\end{aligned}
$$




$$
\begin{gathered}
c_{1}=c_{2}=\left[\begin{array}{cccccccc}
0 & 0.3 & 0.28 & 0.42 & 0 & 0 & 0 & 0 \\
0 & 0 & 0 & 0 & -1.52 & 0 & 2.78 & 0.217 \\
0 & 0 & 0 & 0 & 0 & 0 & 0 & 1
\end{array}\right], \\
d_{3}^{\mathrm{T}}=\left[\begin{array}{llllllll}
0 & 0 & 0 & 0 & 0 & 0 & 0 & 100
\end{array}\right] \\
m_{23}^{\mathrm{T}}=m_{32}^{\mathrm{T}}=\left[\begin{array}{llllllll}
0 & 0 & 0 & 0 & 0 & 0 & 0 & 20.1116
\end{array}\right] \\
m_{13}^{\mathrm{T}}=m_{31}^{\mathrm{T}}=m_{23}^{\mathrm{T}}, \\
b_{3}=\left[\begin{array}{llllllll}
2 & 0 & 0 & 0 & 5 & 0 & 0 & 0
\end{array}\right]^{\mathrm{T}} .
\end{gathered}
$$

Note that $\alpha_{1 i}=1$ when the loads of each area are balanceable. Here, the interconnected items $A_{i j}$ can be written as the form proposed in this paper, namely, $A_{i j}=G_{i} E_{i, j} H_{j}$, where

$$
\begin{aligned}
G_{i} & =\left[\begin{array}{lll}
0 & 0 & m_{i j}^{\mathrm{T}}
\end{array}\right]^{\mathrm{T}}, \\
H_{j} & =\left[\begin{array}{llllllllll}
0 & 0 & 0 & 0 & 0 & 0 & 0 & -1 & 0 & 0
\end{array}\right] .
\end{aligned}
$$

$E_{i, j}$ is the interconnection term in the connective matrix.

Suppose that there are two areas $S_{1}$ and $S_{2}$ in the original structural system, namely, $i, j=1,2$. According to the method described in this paper, the control gains $K_{1}, K_{2}$ and observer gains $L_{1}, L_{2}$ can be designed as follows:

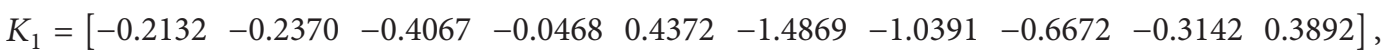

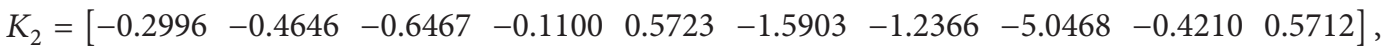

$$
L_{1}=\left[\begin{array}{ccccc}
48.8856 & 0.0233 & -4.0629 & -0.0006 & -0.0006 \\
34.0635 & 0.0198 & -0.0533 & -0.0005 & -0.0004 \\
61.4801 & 0.0293 & -0.0812 & -0.0010 & -0.0019 \\
17.5001 & 0.0093 & -0.0258 & -0.0003 & -0.0007 \\
-1.1657 & 71.0700 & -50.5970 & -1.6831 & -5.8912 \\
-0.3476 & 26.7390 & -15.8781 & -0.6497 & -2.5302 \\
-0.6940 & 52.6061 & -31.8463 & -1.3029 & -5.0800 \\
0.1586 & 1.4854 & 32.3733 & 4.9883 & 24.0253 \\
0.0016 & 0.0937 & 4.9914 & 32.8082 & 0.4984 \\
0.0025 & 0.3659 & 24.0264 & 0.5016 & 32.8082
\end{array}\right],
$$



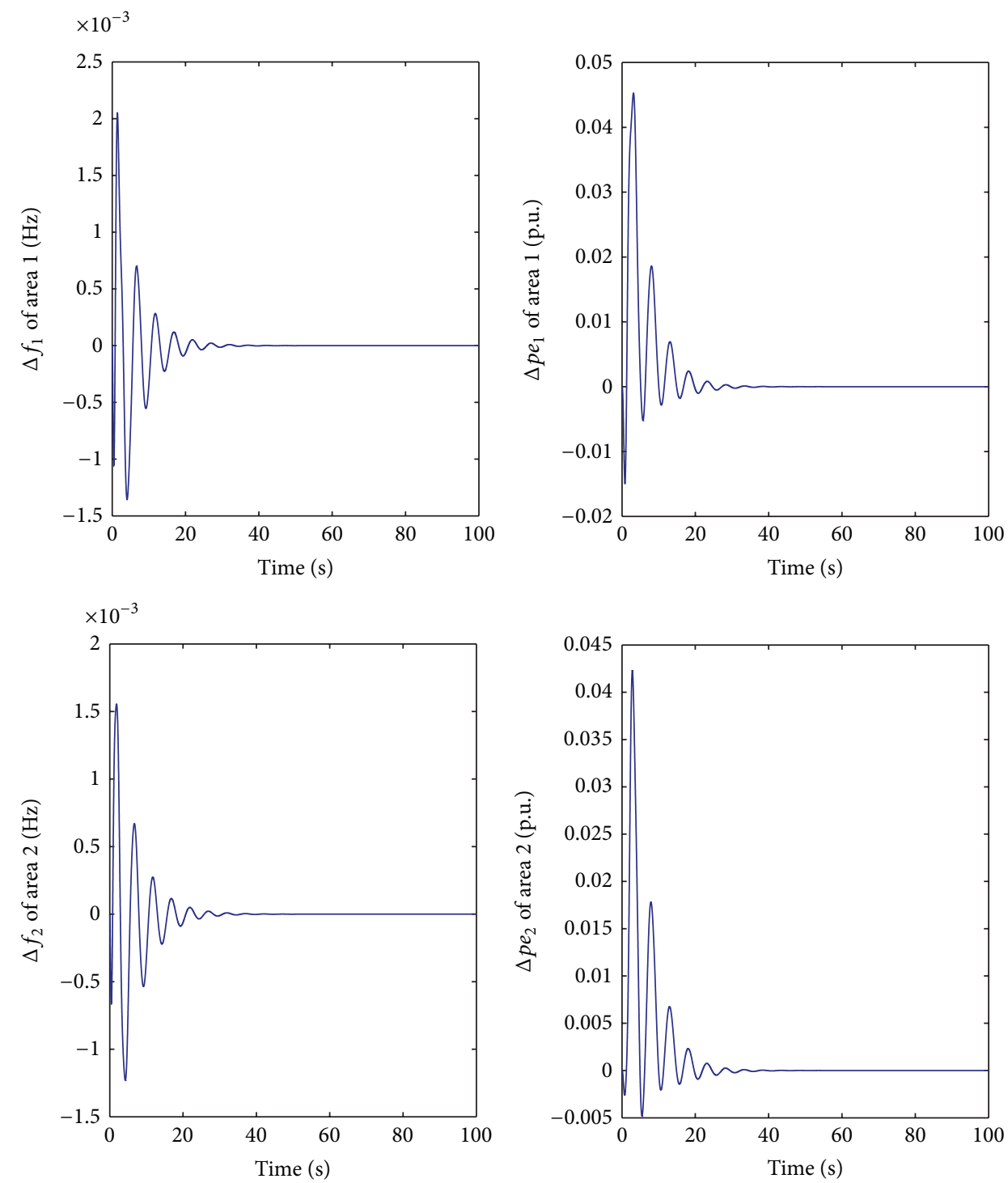

FIGURE 2: Step load disturbance responses of the original subsystems.

The simulation with step load disturbance is shown in Figure 2. The effects of the observers are shown in Figure 3.

In Figure 2, $\Delta f_{i}$ is the frequency variation of each power subsystem, and $\Delta p e_{i}$ is the inversion power variation. When the connection $E_{1,2}$ varies from 1 to 0 , the original system is still stable with step load disturbance and the responses are shown in Figure 4. So the original structural system is connectively stable.

Now, a new subsystem $S_{3}$ is added to the original structural system. There are three cases to be considered.

Case 1. The dimension of the added subsystem is the same as the original subsystems.

Consider the case that a new subsystem $S_{3}$ of the same dimensionality as the original subsystems is added to the original structure composed of the two subsystems. According to the method described above, we choose $q_{i}(i=1,2,3)$ as

$$
q_{i}=\left[\begin{array}{cccccccccc}
0 & 0 & 0 & 0 & 0 & 0 & 0 & 0 & 0 & 0 \\
0 & 0 & 0 & 0 & 0 & 0 & 0 & 0 & 0 & 0 \\
0 & 0 & 0 & 0 & 0 & 0 & 0 & 0 & 0 & 0 \\
0 & 0 & 0 & 0 & 0 & 0 & 0 & 0 & 0 & 0 \\
0 & 0 & 0 & 0 & 0 & 0 & 0 & 0 & 0 & 0 \\
0 & 0 & 0 & 0 & 0 & 0 & 0 & 0 & 0 & 0 \\
0 & 0 & 0 & 0 & 0 & 0 & 0 & 0 & 0 & 0 \\
0 & 0 & 0 & 0 & 0 & 0 & 0 & 0 & 0 & 0 \\
0 & 0 & 0 & 0 & 0 & 0 & 0 & 0 & 0 & 0 \\
0 & 0 & 0 & 0 & 0 & 0 & 0 & -1 & 0 & 0
\end{array}\right]
$$



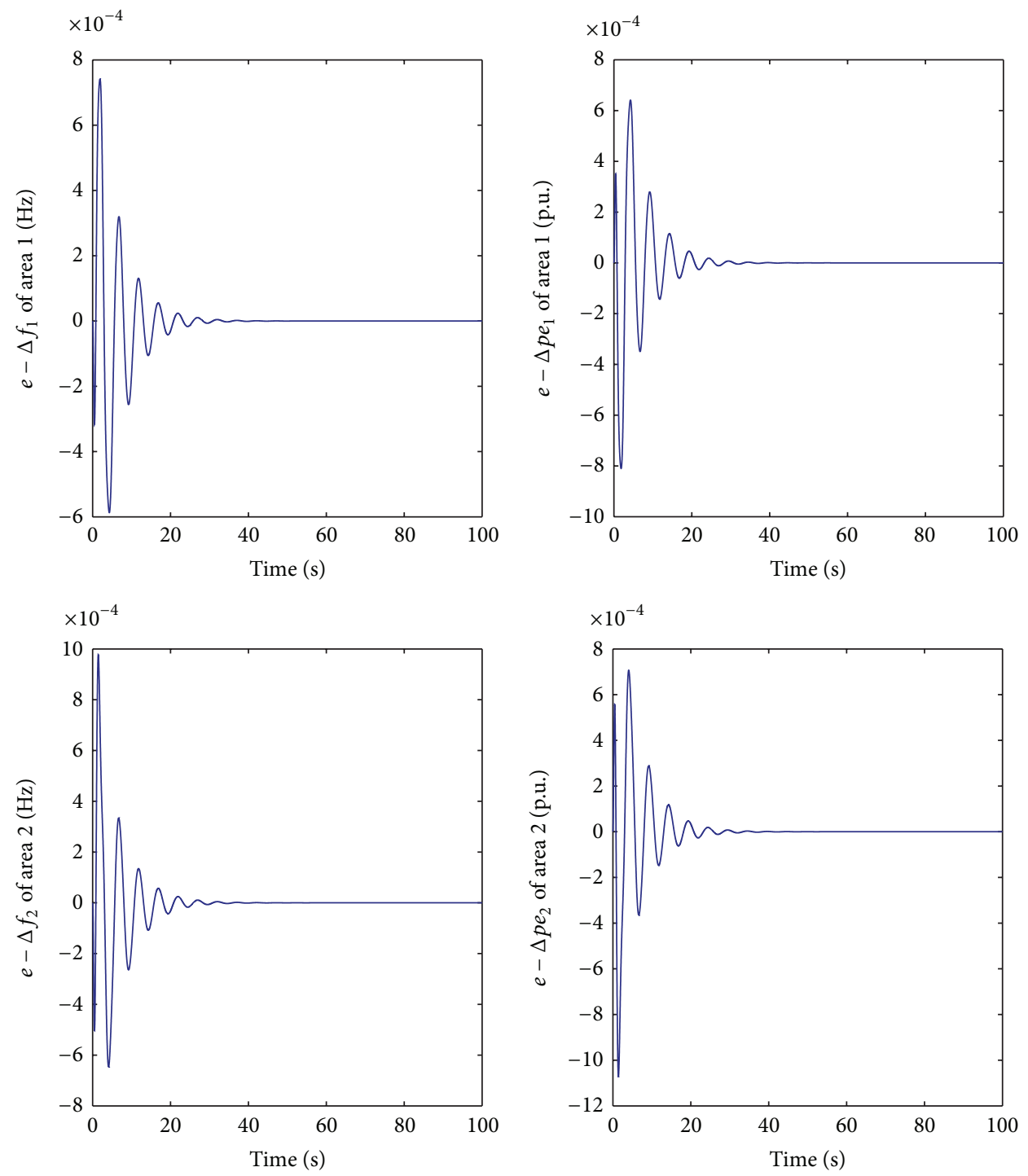

FIgURE 3: Observation errors of the original subsystems.

and substitute the parameters of $S_{3}$ into the linear matrix inequality (13) to find a solution. According to (15), the control law $K_{3}$ and the observer gain $L_{3}$ of the new subsystem can be obtained as

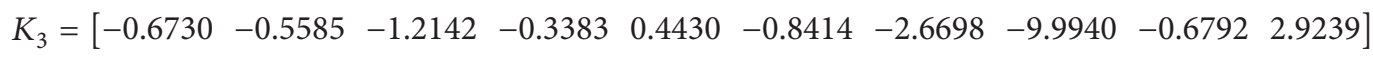

$$
\begin{aligned}
& L_{3}=\left[\begin{array}{ccccc}
59.1012 & 0.0275 & -4.0513 & -0.0012 & -0.0006 \\
40.3382 & 0.0223 & -0.0419 & -0.0013 & -0.0007 \\
79.0068 & 0.0370 & -0.0667 & 0.0007 & 0.0006 \\
22.8802 & 0.0116 & -0.0209 & 0.0003 & 0.0002 \\
-1.6962 & 105.4925 & -63.4223 & -21.8307 & -10.0700 \\
-0.5370 & 40.7204 & -21.2093 & -8.5926 & -4.2251 \\
-0.9949 & 75.0621 & -39.5195 & -15.9553 & -7.8495 \\
0.1396 & 1.4414 & 41.3652 & 49.8938 & 30.0730 \\
0.0002 & 0.9801 & 49.8935 & 41.7897 & 0.5000 \\
-0.0001 & 0.4827 & 30.0731 & 0.5000 & 41.7897
\end{array}\right]
\end{aligned}
$$



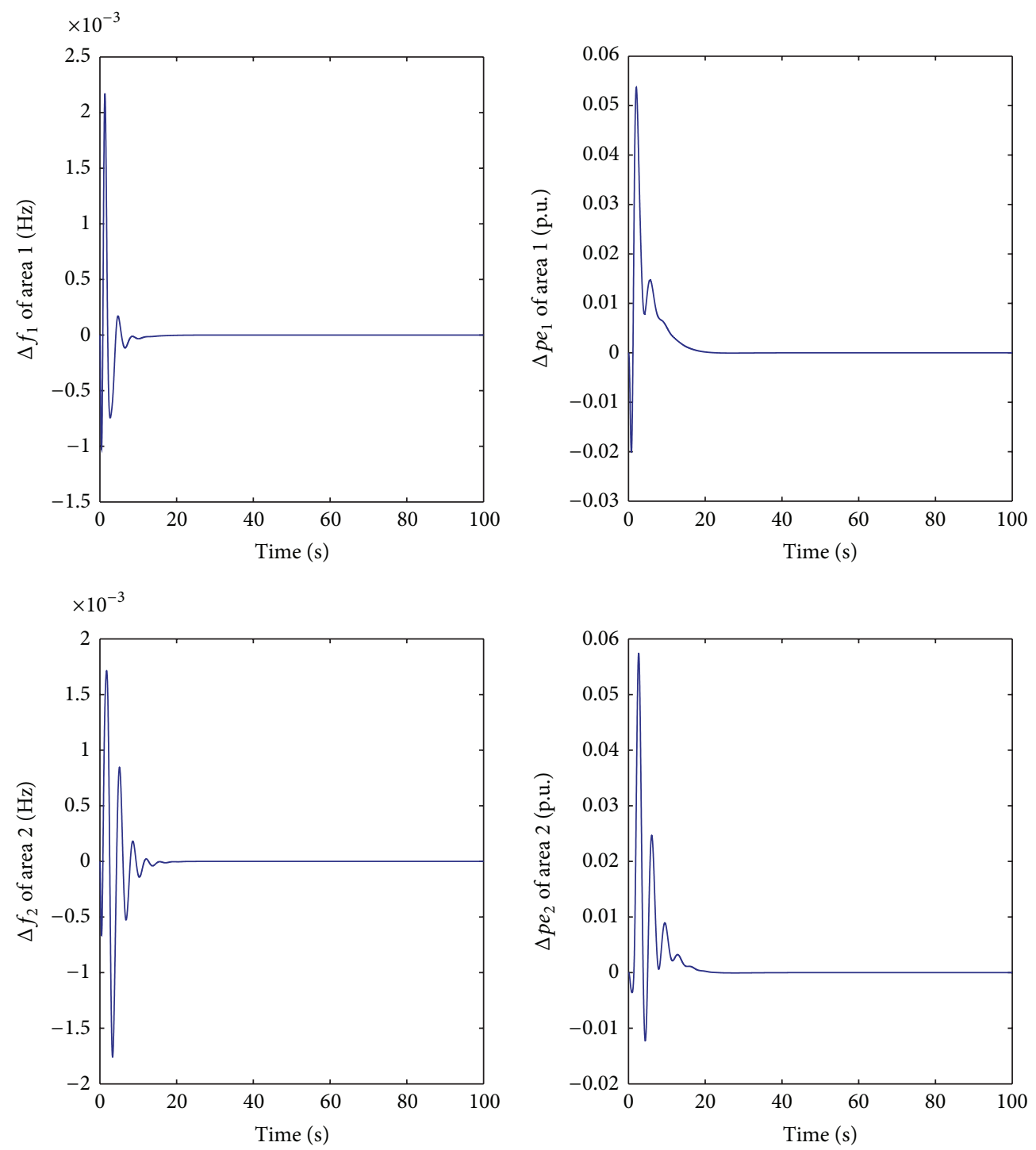

FIGURE 4: Step load disturbance responses of the original subsystems when $E_{1,2}$ varies from 1 to 0 .

Then the simulation of the whole system with step perturbation is performed and the results are shown in Figures 5 and 6 . From these figures, it can be seen that both the new subsystem and the original structure system are stable and the state observation errors converge to zero.

In order to check the connective stability of the expanded system, we cut off one of the interconnections between the subsystems. For example, the connection $E_{1,3}$ or $E_{2,3}$ is varied from 1 to 0 and the simulation on the system with step disturbance is repeated. The results are shown in Figures 7 and 8.

From Figures 5, 6, 7, and 8, we can see that the system is still stable when the interconnection between the subsystems is cut off, as the response curves have not basically changed. Consequently the overall system is robustly connectively stable with strong robustness. The results illustrate that the proposed method can guarantee the steady operation of the interconnected power system and also meet the requirements of decentralized load frequency control of power systems.

Case 2. The dimension of the added subsystem is different from the original subsystems.

The newly added subsystem $S_{3}$ is a six-dimensional system with the form in (30). The matrices of the subsystem can be found in [17]. 

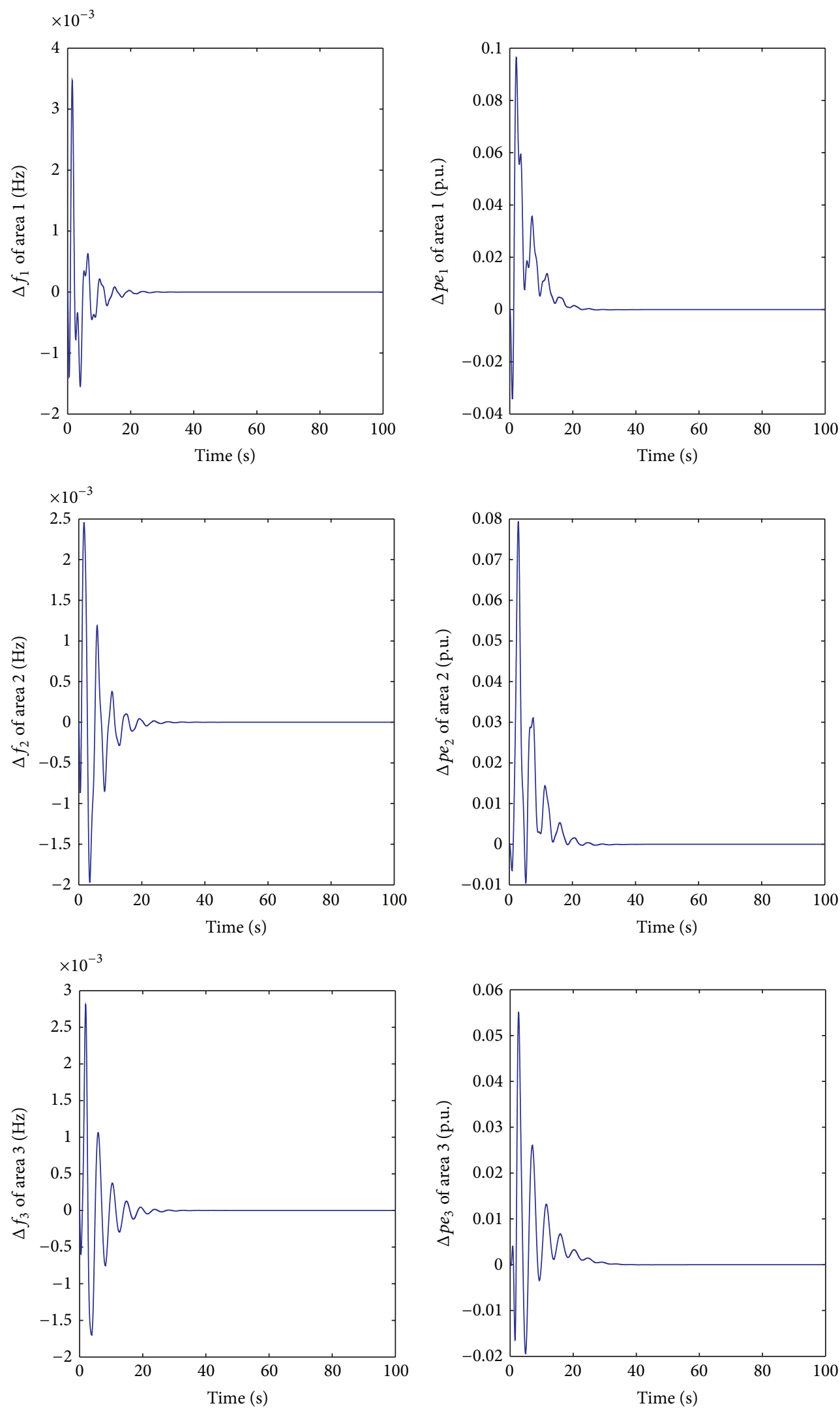

FIGURE 5: Step load disturbance responses of the original subsystems and the newly added subsystem. 

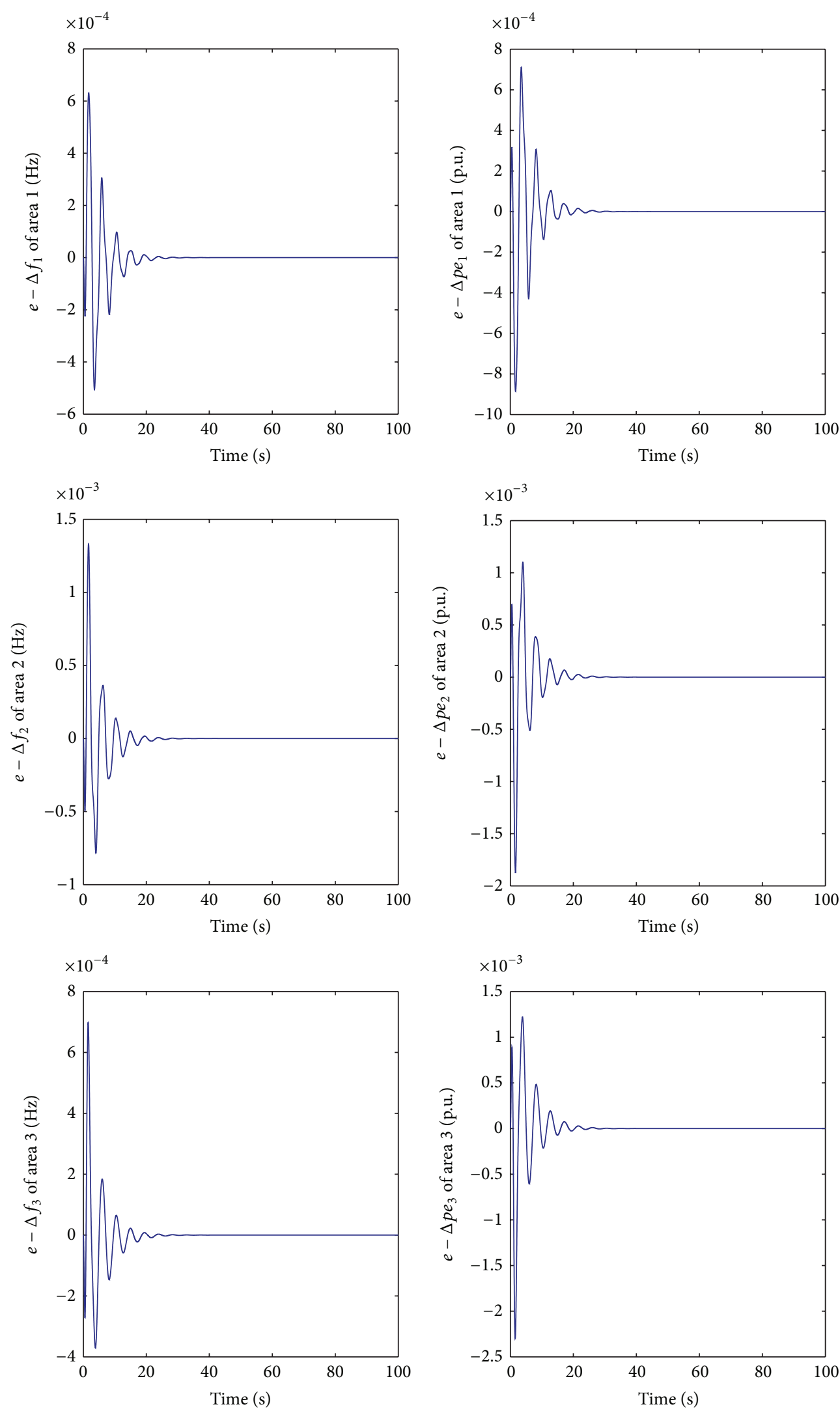

FIGURE 6: Observation errors of the original subsystems and the newly added subsystem. 

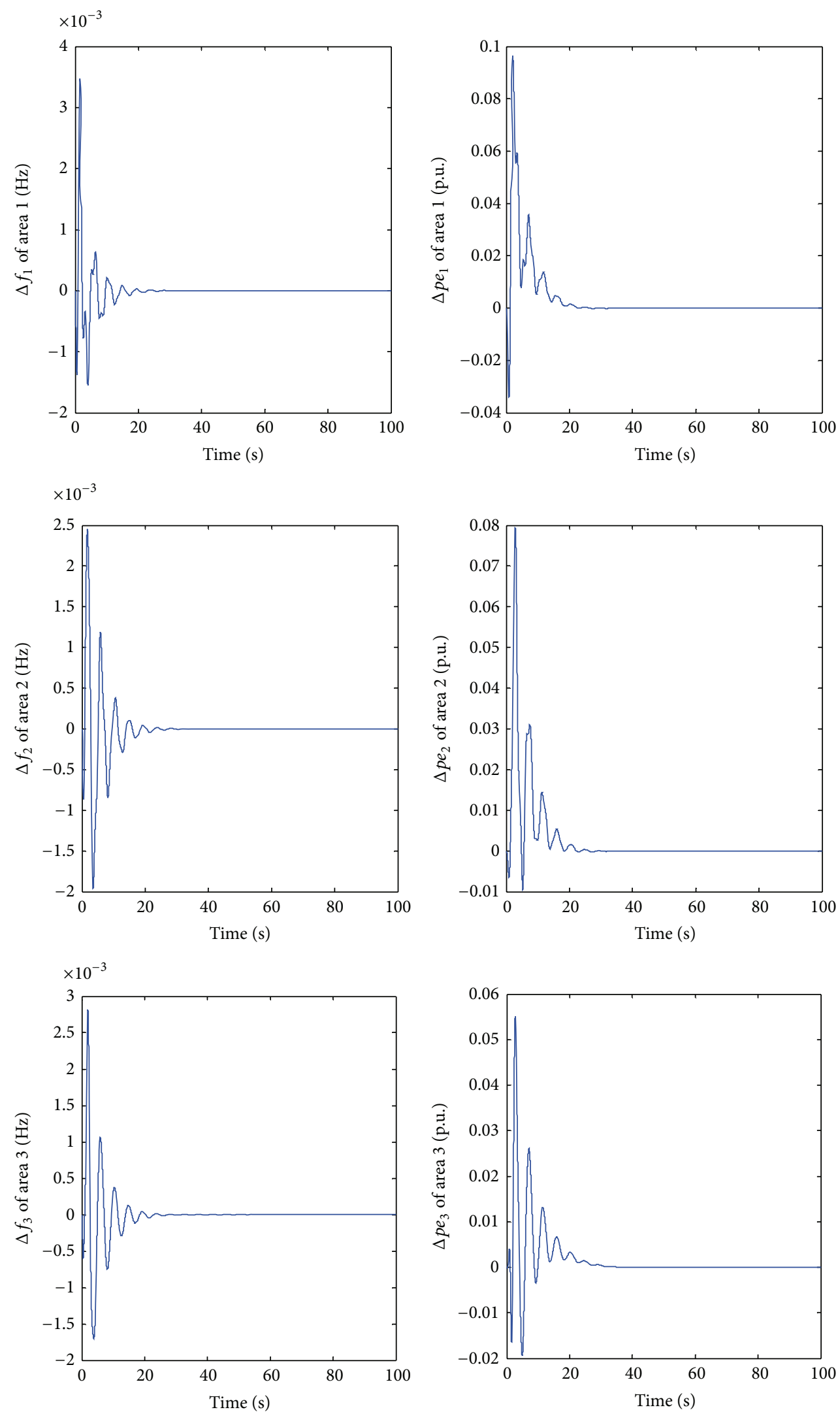

FIgURE 7: Step responses of the expanded system when $E_{1,3}$ varies from 1 to 0. 

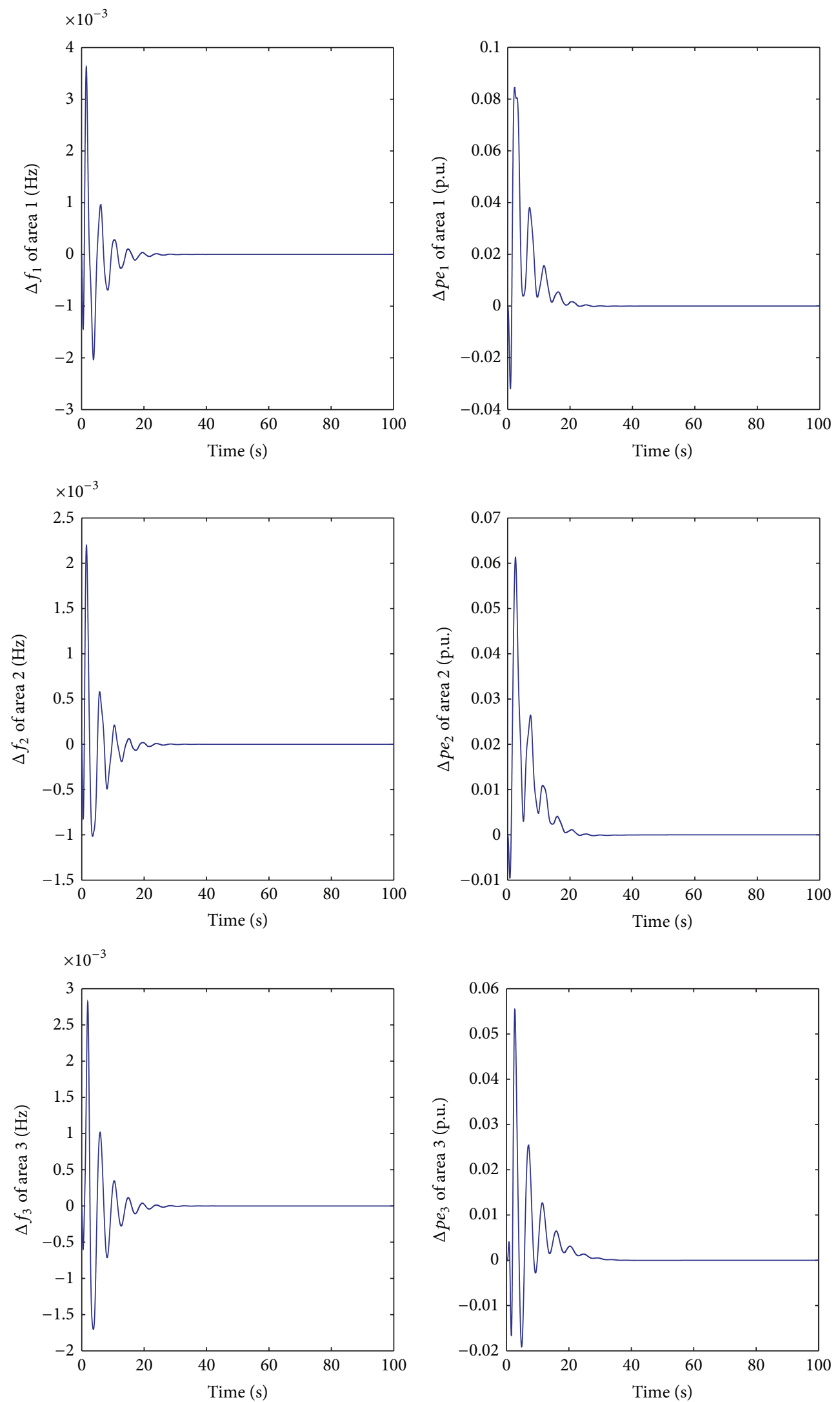

FIgURE 8: Step responses of the expanded system when $E_{2,3}$ varies from 1 to 0. 

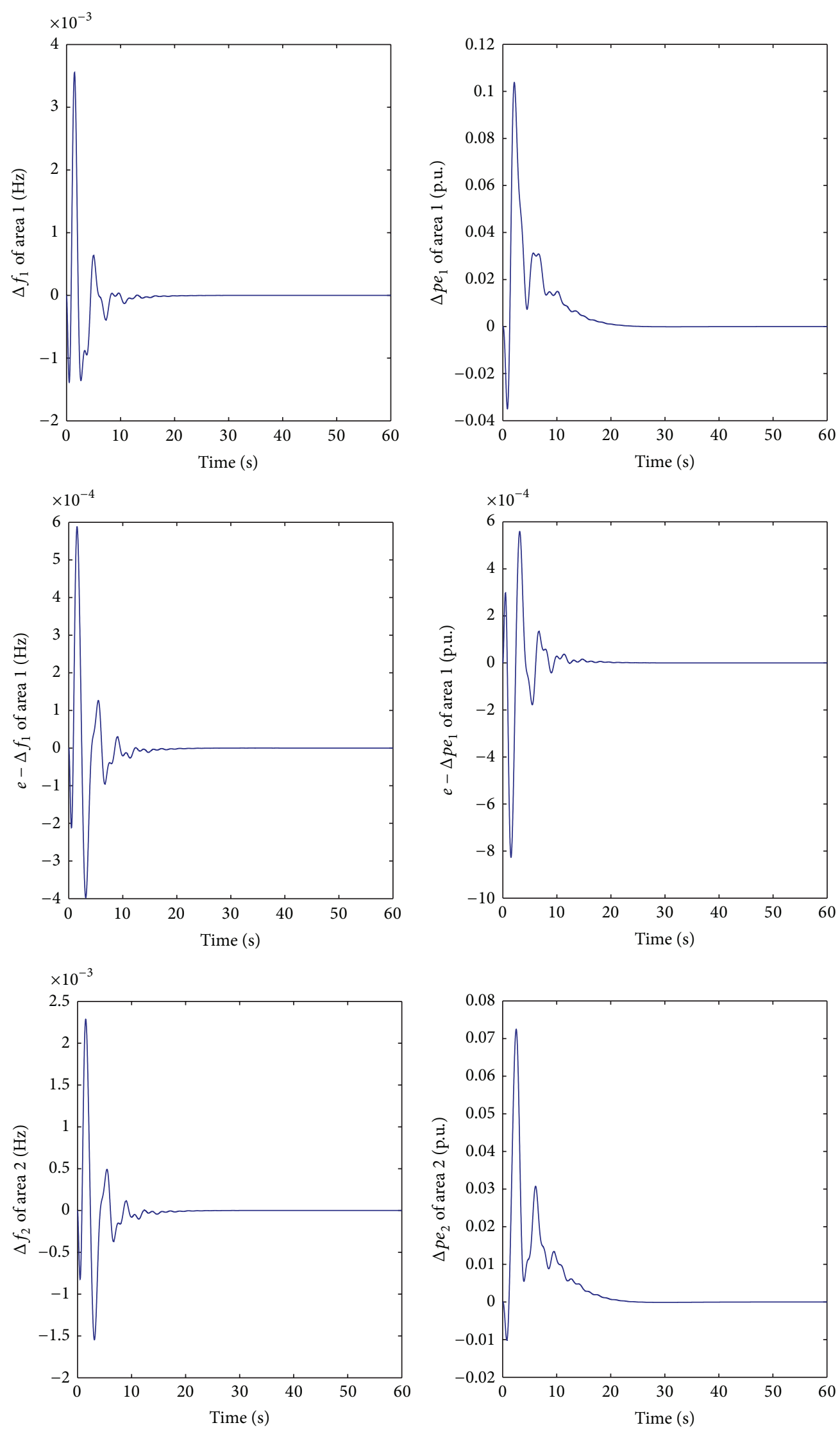

(a)

FIgURE 9: Continued. 

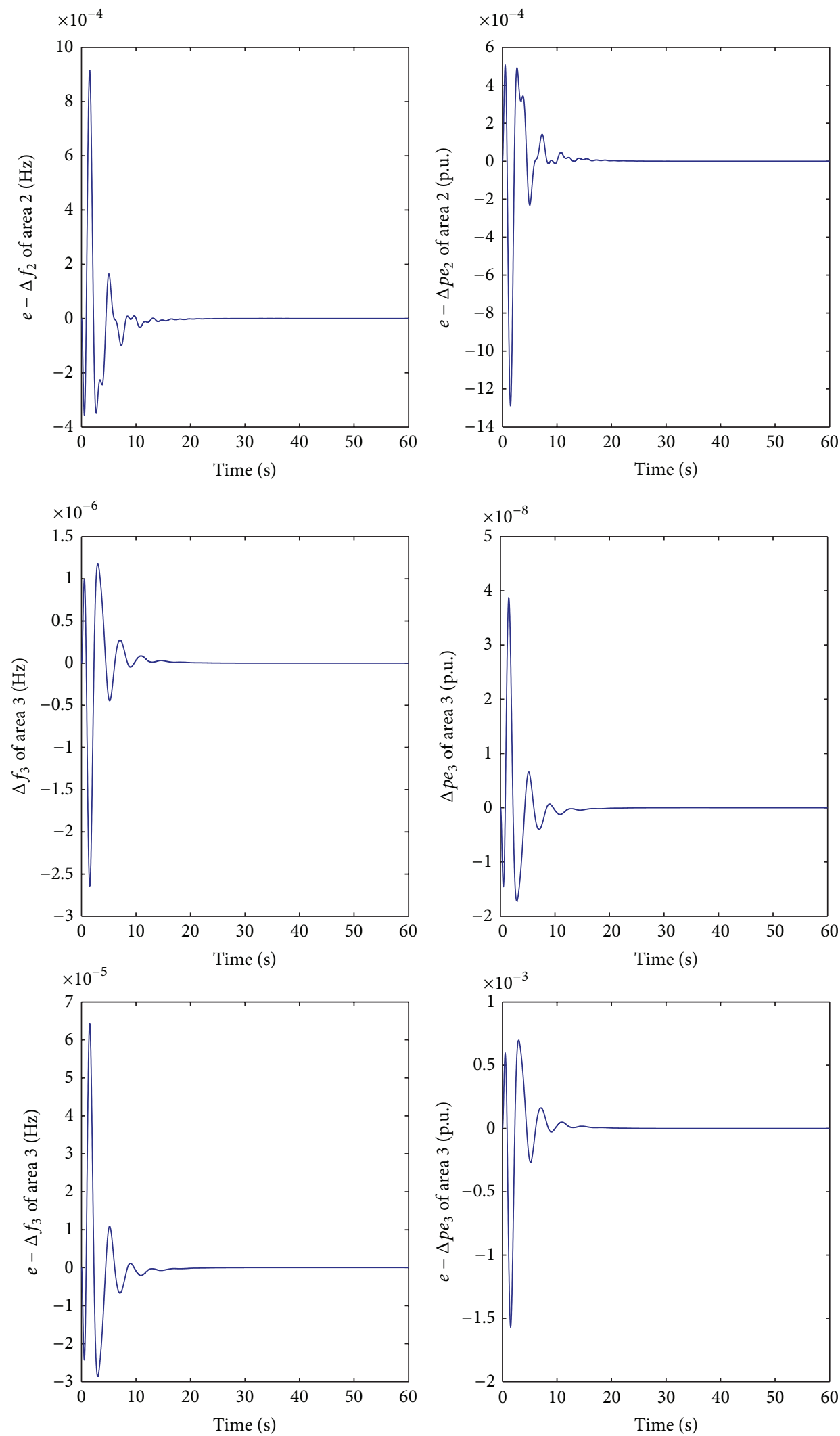

(b)

FIGURE 9: Step load disturbance responses and observation errors of the original subsystems and the newly added subsystem. 

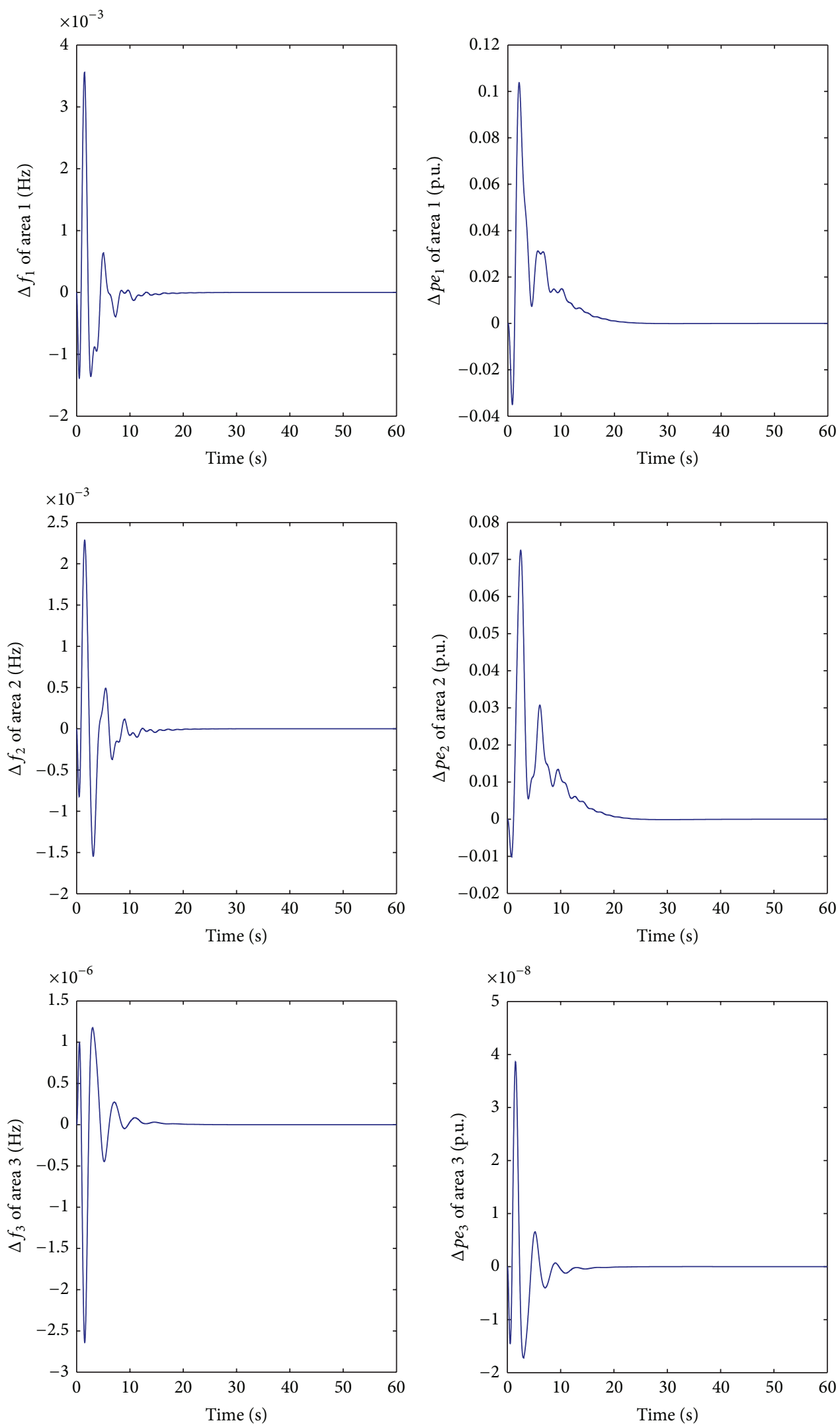

FIGURE 10: Step responses of the expanded system when $E_{1,3}$ varies from 1 to 0 . 

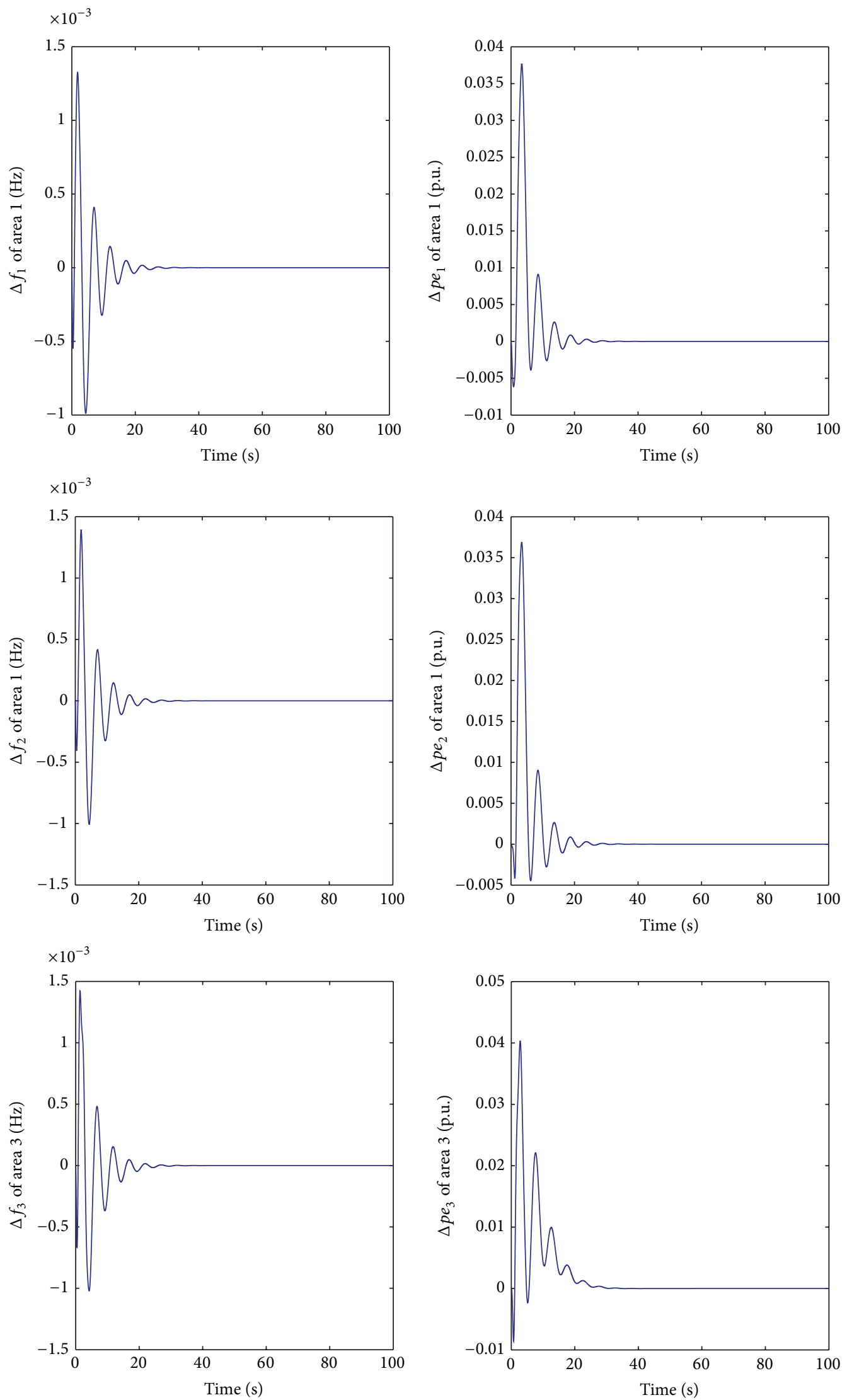

(a)

FIGURE 11: Continued. 

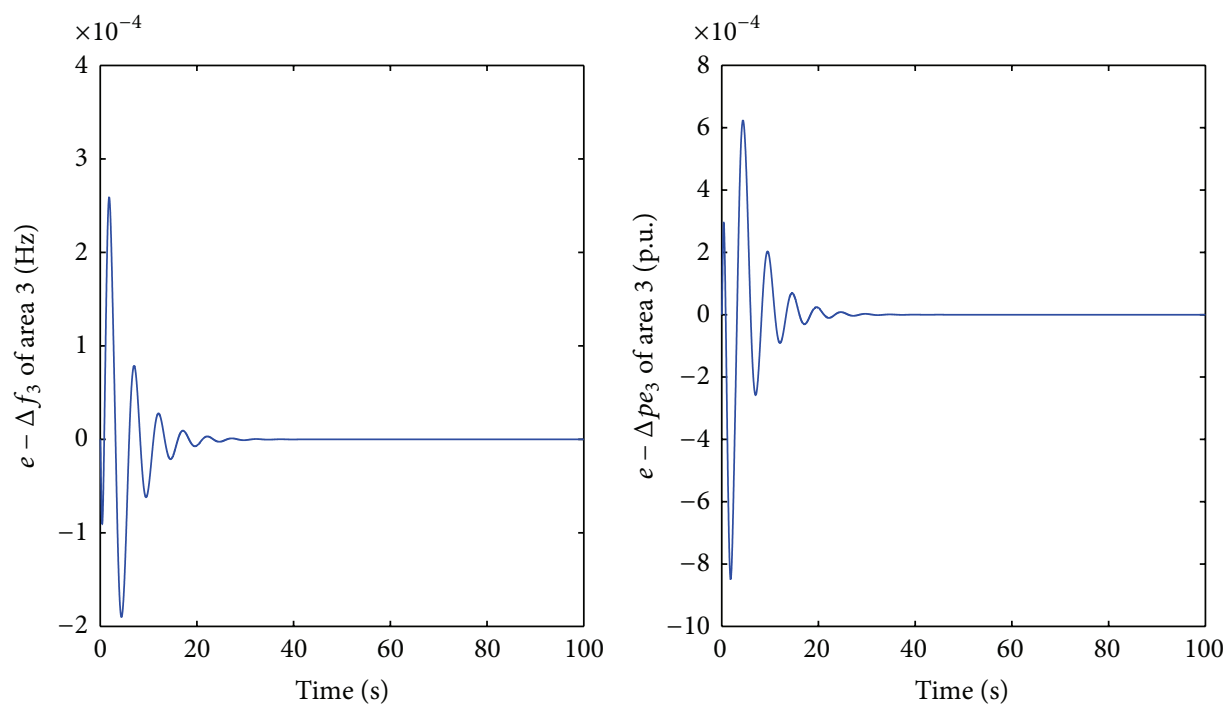

(b)

FIGURE 11: Step load disturbance responses and observation errors of the expanded system when two original-construction subsystems are designed by LQG approach.

According to the method described in this paper, by choosing $q_{i}(i=1,2,3)$ properly, the simulation on the whole system with step perturbation is performed. The simulation results are shown in Figure 9. When the connection $E_{1,3}$ is varied from 1 to 0 , the simulation with step disturbance is repeated, as shown in Figure 10.

From these figures, it can be seen that both the new subsystem and the original subsystems are stable and connectively stable, and state observation errors are able to converge to zero. So the proposed method is effective.

Case 3. The subsystems in original structure have different controllers from the one in this paper.

In this paper, we have studied that the control design approach for original-construction subsystems is the same as the newly added subsystem, but the situation is often otherwise. In practice, the controllers for the original-construction subsystems were often designed before a new subsystem is added, and the design method employed may be different from the method presented in this paper. To illustrate the applicability of the proposed method, the simulation results are given for the case that the two original-construction subsystems are controlled by LQG approach and the newly added subsystem is controlled by the present method. The step responses of the load disturbance are shown in Figure 11. In order to check the connective stability of the expanded system, the connection $E_{2,3}$ is cut off with the step disturbance and the simulation results are shown in Figure 12.

From these figures, we can see that the method presented in this paper is still effective when the original structure has different controllers from the new subsystem. The overall system is still connectively stable and has strong robustness. The requirements of decentralized load frequency control of power systems are met as well.

\section{Conclusion}

An organically structured control problem based on state observation for a class of large-scale systems with expanding construction has been studied in this paper. Making use of stability theory, LMI approach, and split matrix algorithms, the sufficient condition for robust decentralized connective stabilization with observers has been derived for a class of large-scale systems with expanding construction. A design method for the controller and observer has been proposed. This method can be used to design the decentralized control law and the observer gain matrix of a new subsystem without changing the control laws of the original structural systems, so that the new subsystem with observer and the whole expanded system are all connectively stable. The results have been applied to the expansion of a class of interconnected power systems. The simulation results show the effectiveness of this method, and the resulting system has good robustness. Since the proposed decentralized control design method in this paper considers simultaneously the robustness for system structure and the connective stability on the basis of not changing the decentralized state feedback control laws of the original construction, this paper can be used as the theoretical basis for expansion of interconnected large-scale system online.

\section{Conflict of Interests}

The authors declare that there is no conflict of interests regarding the publication of this paper.

\section{Acknowledgments}

This work is supported by the National Natural Science Foundation of China (no. 61273011). The authors of this paper 

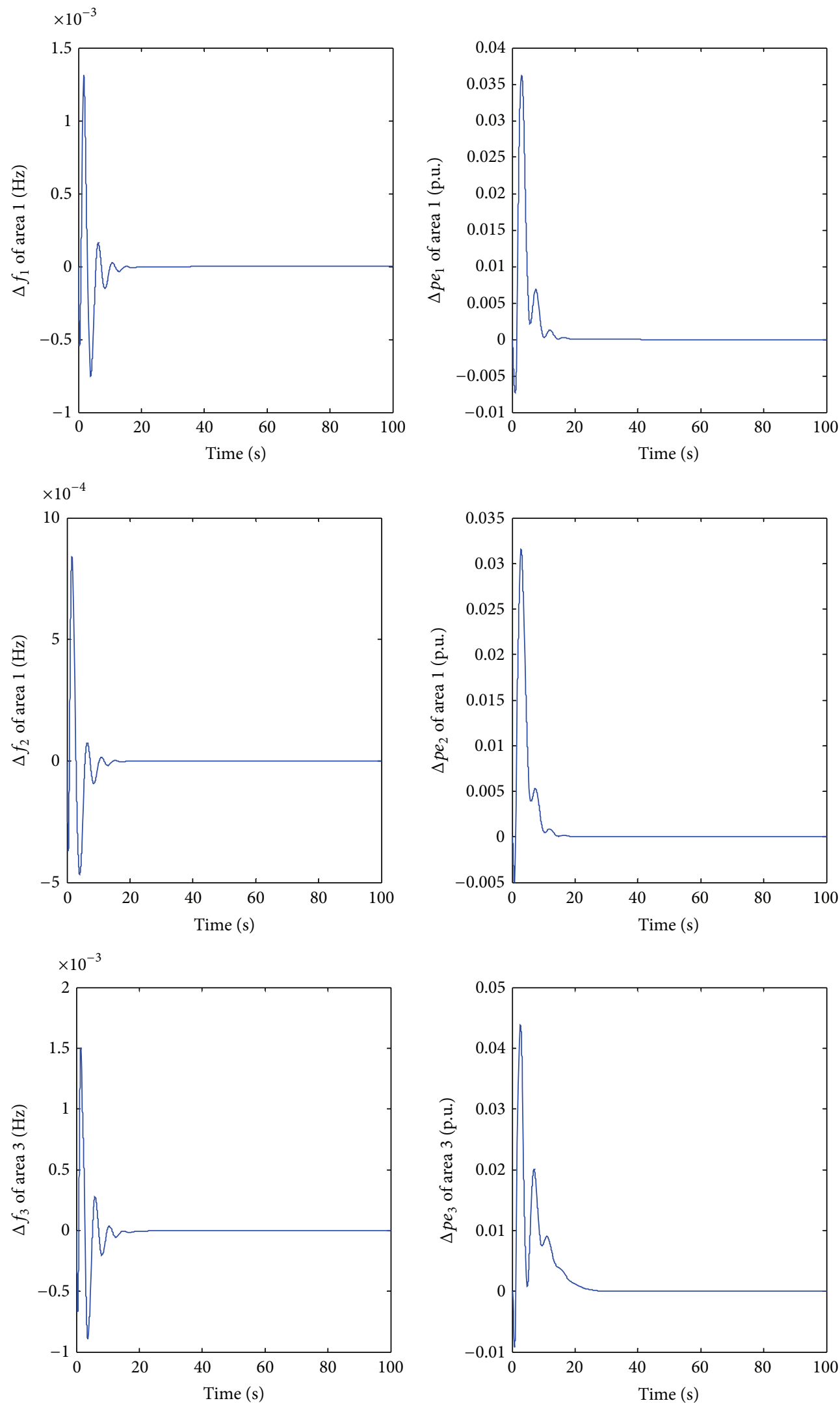

FIGURE 12: Step responses and observation errors of the expanded system when two original-construction subsystems are designed by LQG approach and $E_{2,3}$ varies from 1 to 0 . 
would like to express gratitude to Matthew MacDonald for his contributions to the editing of this paper.

\section{References}

[1] D. D. Siljak, Large-Scale Dynamic Systems: Stability and Structure, North-Holland, New York, NY, USA, 1978.

[2] D. D. Šiljak and D. M. Stipanović, "Organically-structured control," in Proceedings of the American Control Conference, pp. 2736-2742, Arlington, Va, USA, June 2001.

[3] N. Gattie, "A connective stability analysis of complex system simulation and control via multi-agent systems," in Proceedings of the 2nd Starting AI Researchers' Symposium, pp. 26-37, 2004.

[4] S.-L. Sun, P. Peng, and C.-T. Chen, "Connective stability of a kind of singular nonlinear large-scale dynamical systems," in Proceedings of the IEEE International Symposium on Industrial Electronics (ISIE '09), pp. 1731-1736, Seoul, South Korea, July 2009.

[5] X.-L. Tan and M. Ikeda, "Decentralized stabilization for expanding construction of large-scale systems," IEEE Transactions on Automatic Control, vol. 35, no. 6, pp. 644-651, 1990.

[6] X. H. Li, Y. W. Jing, S. C. Xu, and L. J. Wu, "LMI approach of decentralized connective stabilization for expanded structure of large scale systems," Journal of Northeastern University (Natural Science), vol. 28, pp. 1073-1080, 2007 (Chinese).

[7] X. Li, H. Guo, and Y. Jing, "Robust decentralized connective stabilization for expanding construction of large-scale systems," in Proceedings of the IEEE International Conference on Automation and Logistics (ICAL '08), pp. 1121-1125, IEEE, Qingdao, China, September 2008.

[8] A. Arunkumar, R. Sakthivel, K. Mathiyalagan, and S. M. Anthoni, "State estimation for switched discrete-time stochastic BAM neural networks with time varying delay," Nonlinear Dynamics, vol. 73, no. 3, pp. 1565-1585, 2013.

[9] S. Lakshmanan, K. Mathiyalagan, J. H. Park, R. Sakthivel, and F. A. Rihan, "Delay-dependent $H_{\infty}$ state estimation of neural networks with mixed time-varying delays," Neurocomputing, vol. 129, pp. 392-400, 2014.

[10] S. S. Stanković, D. M. Stipanović, and D. D. Šiljak, "Decentralized dynamic output feedback for robust stabilization of a class of nonlinear interconnected systems," Automatica, vol. 43, no. 5, pp. 861-867, 2007.

[11] A. S. Tlili and N. B. Braiek, "Systematic linear matrix inequality conditions to design a robust decentralised observer-based optimal control for interconnected systems," IET Control Theory \& Applications, vol. 6, no. 18, pp. 2737-2747, 2012.

[12] M. Mansouri, L. Boutat-Baddas, M. Darouach, and H. Messaoud, "Decentralized observers for a class of large-scale singular systems via LMI," International Journal of Intelligent Computing and Cybernetics, vol. 6, no. 2, pp. 158-181, 2013.

[13] S. Sui, S. Tong, and Y. Li, "Observer-based adaptive fuzzy decentralized control for stochastic large-scale nonlinear systems with unknown dead-zones," Information Sciences, vol. 259, pp. 71-86, 2014.

[14] X. Li and Y. Huang, "Organically-structured control of largescale systems with expanded construction based on state observer," in Proceedings of the 24th Chinese Control and Decision Conference (CCDC '12), pp. 488-492, May 2012.

[15] M. S. Calovic, M. V. Djorovic, and D. D. Siljak, "Decentralized approach to automatic generation control of interconnected power systems," in Proceedings of the International Conference on Large High-Voltage Electric System, pp. 1-12, 1978.
[16] X. B. Chen, Inclusion Principle and Its Applications, 2012.

[17] H. L. Zeynelgil, A. Demiroren, and N. S. Sengor, "The application of ANN technique to automatic generation control for multi-area power system," International Journal of Electrical Power and Energy Systems, vol. 24, no. 5, pp. 345-354, 2002. 


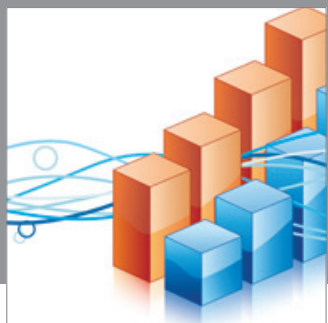

Advances in

Operations Research

mansans

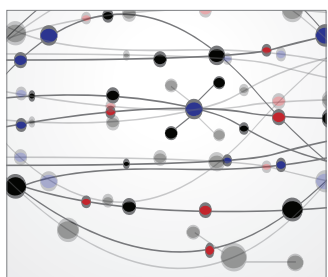

The Scientific World Journal
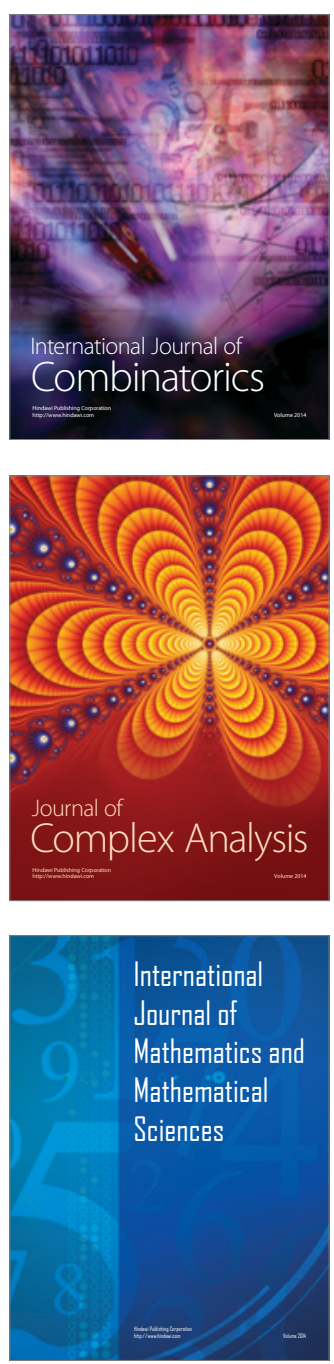
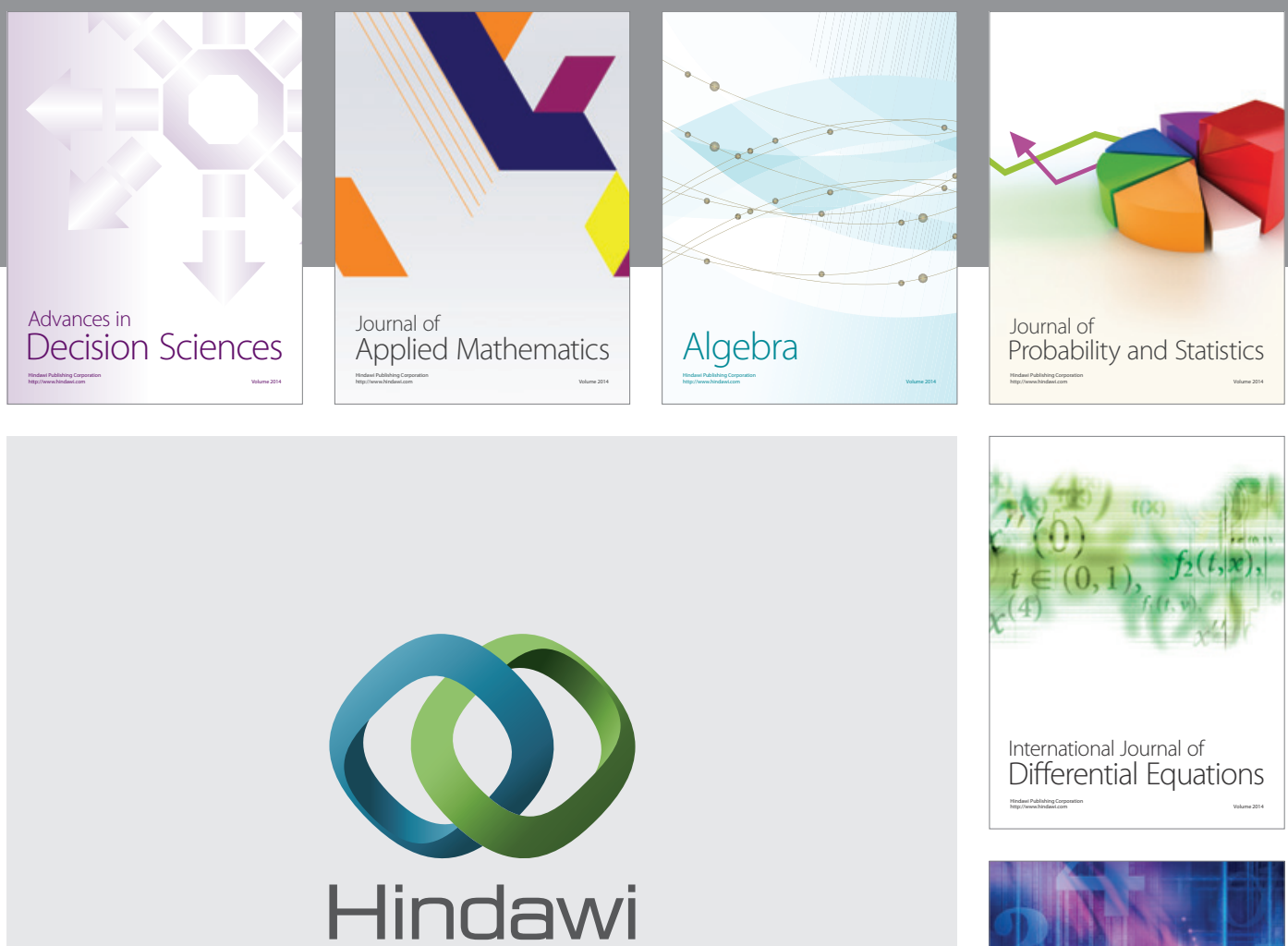

Submit your manuscripts at http://www.hindawi.com
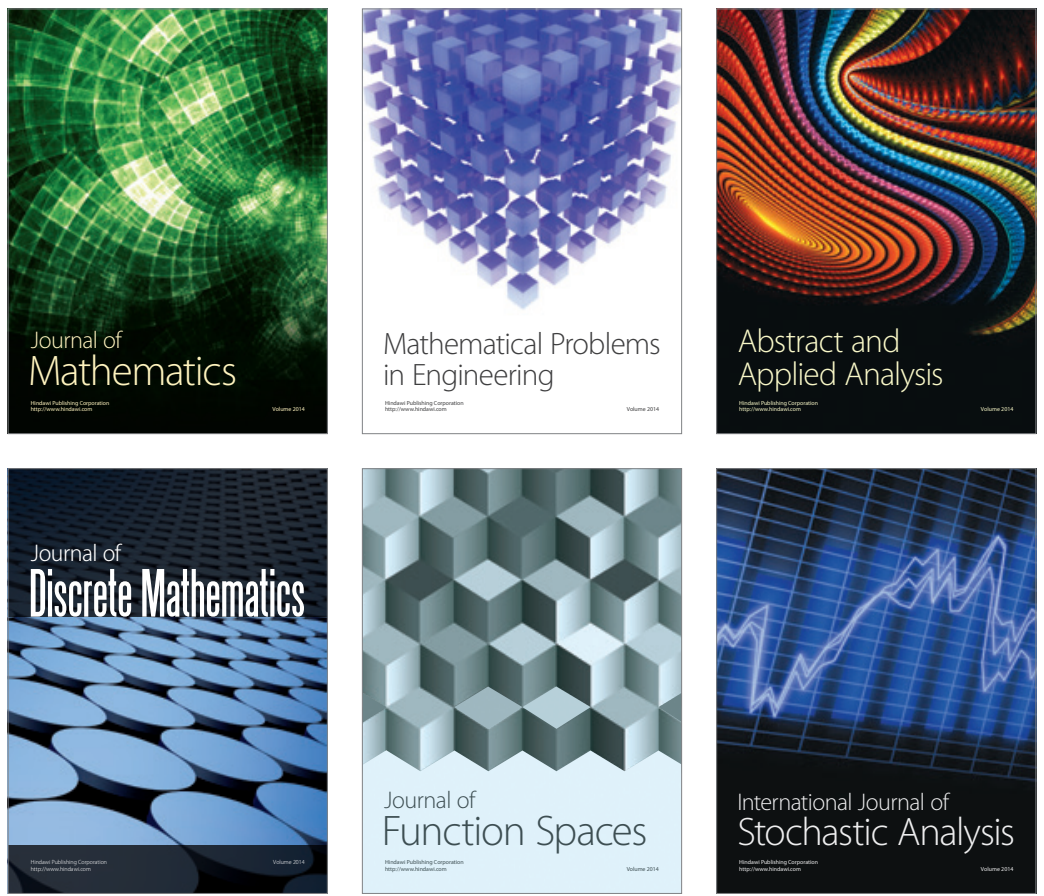

Journal of

Function Spaces

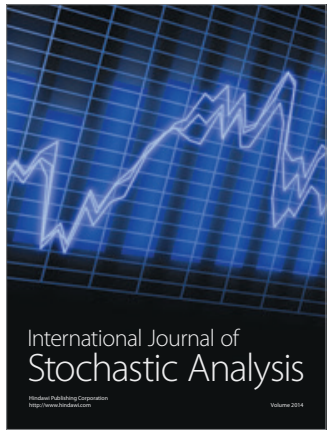

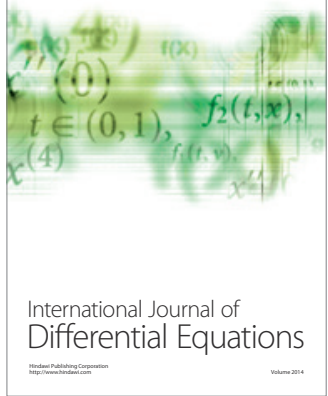
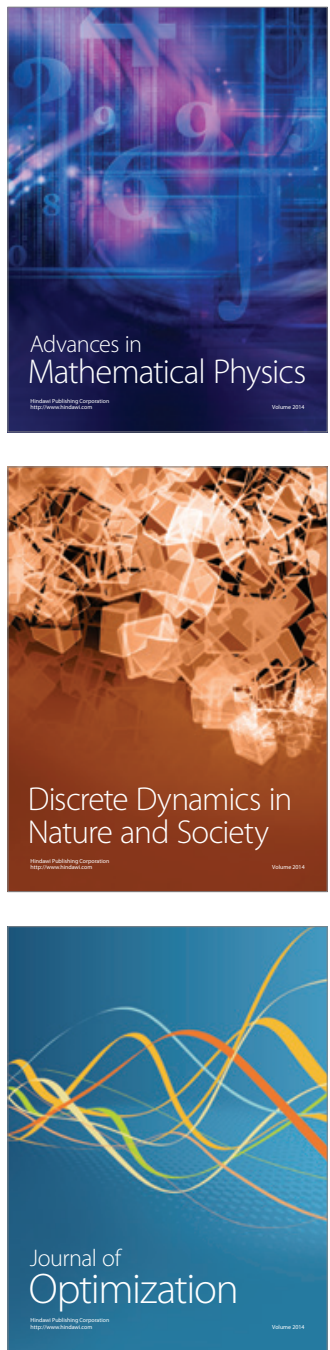